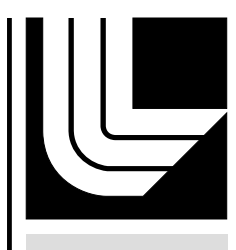

LAW RENCE LIVERMORE N A TION A L LABORATORY

Collapsing Bubble in Metal for High Energy Density Physics Study

S. F. Ng, J. J. Barnard, P. T. Leung, S. S. Yu

April 14, 2011

High Energy Density Physics 
This document was prepared as an account of work sponsored by an agency of the United States government. Neither the United States government nor Lawrence Livermore National Security, LLC, nor any of their employees makes any warranty, expressed or implied, or assumes any legal liability or responsibility for the accuracy, completeness, or usefulness of any information, apparatus, product, or process disclosed, or represents that its use would not infringe privately owned rights. Reference herein to any specific commercial product, process, or service by trade name, trademark, manufacturer, or otherwise does not necessarily constitute or imply its endorsement, recommendation, or favoring by the United States government or Lawrence Livermore National Security, LLC. The views and opinions of authors expressed herein do not necessarily state or reflect those of the United States government or Lawrence Livermore National Security, LLC, and shall not be used for advertising or product endorsement purposes. 


\title{
Collapsing Bubble in Metal for High Energy Density Physics Study
}

\author{
Siu-Fai Ng ${ }^{1,2}$, J. J. Barnard ${ }^{3}$, P. T. Leung ${ }^{1 *}$ and S. S. Yu ${ }^{1,2}$ \\ ${ }^{1}$ Department of Physics and Institute of Theoretical Physics, \\ The Chinese University of Hong Kong, Hong Kong SAR, China \\ ${ }^{2}$ Lawrence Berkeley National Laboratory, Berkeley, CA94720, USA \\ ${ }^{3}$ Lawrence Livermore National Laboratory, Livermore, CA94550, USA
}

(Dated: April 6, 2011)

\begin{abstract}
This paper presents a new idea to produce matter in the high energy density physics (HEDP) regime in the laboratory using an intense ion beam. A gas bubble created inside a solid metal may collapse by driving it with an intense ion beam. The melted metal will compress the gas bubble and supply extra energy to it. Simulations show that the spherical implosion ratio can be about 5 and at the stagnation point, the maximum density, temperature and pressure inside the gas bubble can go up to nearly 2 times solid density, $10 \mathrm{eV}$ and a few megabar (Mbar) respectively. The proposed experiment is the first to permit access into the Mbar regime with existing or near-term ion facilities, and opens up possibilities for new physics gained through careful comparisons of simulations with measurements of quantities like stagnation radius, peak temperature and peak pressure at the metal wall.

PACS numbers: 28.52.Av, 41.75.Ak, 78.60.Mq, 64.10.+h
\end{abstract}

\section{INTRODUCTION}

High energy density physics (HEDP) is a study of matter under extreme conditions of pressure, temperature and density. It is an expanding field rich in new physics phenomena and compelling applications. HEDP is generally defined to be the regime with energy densities exceeding $10^{11}$ Joules per cubic meter $\left(\mathrm{J} / \mathrm{m}^{3}\right)$, or pressures exceeding 1 megabar (Mbar) [1].

In general, matter at HEDP conditions has physical properties vastly different from those of matter under ordinary conditions. Understanding of these properties is very important for the study of physics involving dense and strongly coupled plasma, for example, within the interiors of giant planets, low-mass stars, and other astrophysical objects. It is also essential for the study of inertial fusion.

HEDP experiments require deposition of large amounts of energy into a small target volume over a short time interval, while maintaining uniformity across the whole target. To generate matter under high energy density condition in the laboratory, intense ion beams are excellent tools [2-4]. The deposition of ion beams to the target is a well understood process and ion beam heating, in contrast to laser induced heating, is known to be much more uniform across the target over a wide range of thickness from a few microns to a few centimeters, depending on the kinds of beam and target.

There are several such ion beam accelerators for high energy density physics research available, or planned to be built, such as GSI in Germany [5] or NDCX-II in Berkeley [6]. These machines can directly heat the target to temperature of a few $\mathrm{eV}$. The proposed bubble collapse experiment can generate HEDP matter at much higher temperature and pressure than those using the intense ion beam in a simple planar target .

The simplest type of target for creation and study of high energy density states using ion beams uses planar geometry, whereby an ion beam volumetrically heats a target and the target material response is studied via its subsequent radiation and motion. For low energy beams $(\sim 1 \mathrm{MeV}$ per nucleon), a beam illuminates the target normally (see e.g. Ref. [3]), whereas for high energy ion beams $(\sim 1 \mathrm{GeV}$ per nucleon) the planar target may be illuminated in a direction parallel to its face and subsequent expansion normal to the face is detected [7]. Other target geometries, such as cylindrical or spherical, have also been considered and have particular advantages. In Refs. [3] and [8], a simple cylindrical geometry is employed in which a sample is illuminated by a high energy ( $\sim 1 \mathrm{GeV}$ per nucleon) ion beam along the axis of a cylinder and radial expansion is measured (along with other diagnostics). Multilayered cylindrical implosions have also been considered for creation and study of dense plasmas [3, 9, 10]. These targets consist of a mm-scale cylindrical shell of high-Z material that, when isochorically heated by an ion beam, radially compress a sample to create a high density state. The cylindrical geometry enhances the pressure, relative to a planar compression. Spherical geometries have also been considered for ion-beam heated warm dense matter studies as, for example, in Ref. [11], for the purpose of creating small, point-like, radiation sources, or as in Ref. [12] for the purpose

\footnotetext{
* Corresponding author, email:ptleung@phy.cuhk.edu.hk, tel: 852-26096396, fax: 852-26035204
} 
of creating simple uniform samples that are radially tamped and for which the spherical symmetry provides minimal scattering for X-ray diagnostics. Indeed, cylindrical and spherical geometries are employed for inertial confinement fusion targets (see e.g. Refs. [13-15] and references therein) partly because of the enhancement in pressure arising from compression in the non-planar geometry. In this paper, we explore the simplest possible spherically convergent target: an imploding bubble, to explore the extent to which we may create warm dense matter conditions beyond those obtained in planar targets.

The idea of a collapsing bubble is related to the single bubble sonoluminescence (SBSL) experiments [16-22]. SBSL is a phenomenon of periodic light emission by an oscillating micron-sized gas bubble trapped in the pressure anti-node of a standing ultrasound wave with driving pressure of 1.2 to $1.4 \mathrm{~atm}$ in water (or other fluids). It is now strongly believed that the light emission is the consequence of the fast compression of the bubble and the temperature inside the bubble can go up to a few eV [18-22]. So if we use a stronger driving pressure, like a rapidly heated melted metal, the compression may be even stronger and the gas may enter the HEDP regime. That is the basic idea of the proposed experiment.

The proposed experiment considers a gas bubble created within a subrange-thick metal target, as shown in Figure 1. As the target is illuminated by the ion beam, the ion beam energy is deposited into the target. The instantaneous (under constant density) increase in pressure per unit energy deposition per unit mass is:

$$
\left(\frac{\partial P}{\partial E}\right)_{V}=\frac{\alpha K}{c_{v}}
$$

where $P$ is the pressure and $E$ is the energy per unit mass. $\alpha, K$ and $c_{v}$ are the coefficient of thermal expansion, the bulk modulus and the specific heat capacity respectively. Usually, metals and gases have specific heat capacities of the same order of magnitude and metals have very high bulk modului compared to those of gases. Even though metals have relatively low thermal expansion coefficients compared to those of gases, the $(\partial P / \partial E)_{V}$ values of metals are still a factor of a few hundred higher than those of gases. As a result, given a similar energy deposition per atom, the pressure rise in metal is much higher than that inside the gas bubble. If the energy deposition is uniform enough, the bubble will collapse spherically due to the large pressure difference generated. Surface tension may also help to make the bubble collapse spherically. The fast collapse may heat the gas inside the bubble to the high energy density physics regime.

\section{HYDRODYNAMIC SIMULATIONS}

After the intense ion beam is turned on, the ion beam energy will be deposited into the metal, as well as the gas inside the bubble, and the temperature will be raised. The temperature rise will generate a large pressure difference across the bubble wall. To study the proposed experiment quantitatively, hydrodynamic simulations must be done.

As an intense ion beam can have stable beam energy and a long range in the target with uniform beam energy deposition, constant and uniform ion beam energy deposition rate per unit mass to the target and spherical symmetry can be assumed.

A 1-dimension hydrodynamic Lagrangian code for warm dense matter studies in the slab geometry DISH [23] is modified for the spherical geometry in our simulations. For an initially spherical gas bubble, perfect spherical symmetry is assumed in the code. Constant and uniform ion beam energy deposition rate per unit mass to the target is further assumed.

In the modified DISH code, spherical equations are installed. At the boundary between the metal and gas bubble, temperature-dependent surface tension [24] is included:

$$
\sigma=\sigma_{0}\left(1-T / T_{c}\right)^{1.27}
$$

where $T$ and $T_{c}$ are the temperature and the critical temperature respectively. $\sigma_{0}$ is the surface tension at $T=0$. For aluminum, $T_{c} \cong 1 \mathrm{eV}$ and $\sigma_{0}=950 \mathrm{dyn} / \mathrm{cm}$. For temperature higher than the critical temperature, the surface tension vanishes.

For hydrodynamic simulations, especially those with strong shock waves, artificial viscosity has to be implemented so as to obtain realistic results. For our spherical case, Whalen's Tensor Formulation [25] is used in the Lagrangian equations:

$$
\begin{aligned}
\rho \frac{d u}{d t} & =-\frac{d(P+Q)}{d r}-\frac{3 Q}{r} \\
\frac{d E}{d t} & =-(P+Q) \frac{d(1 / \rho)}{d t}+\frac{3 Q u}{r \rho}+\varepsilon_{\mathrm{h}}+\varepsilon_{\mathrm{i}}
\end{aligned}
$$


where $\rho, u, t, P, r, E \varepsilon_{\mathrm{h}}$ and $\varepsilon_{\mathrm{i}}$ are the density, velocity, time, pressure, radius, internal energy per unit mass, heat conduction rate per unit mass and ion energy deposition rate per unit mass. $Q$ is the artificial viscosity term. For a expanding fluid, i.e. $(\Delta u / \Delta r) \geq 0, Q=0$. For a compressing fluid, $Q$ is given by:

$$
Q=c_{0} \rho \frac{d u}{d r}\left(\frac{d u}{d r}-\frac{u}{r}\right) \Delta r^{2}
$$

where $c_{0}$ is a dimensionless viscosity coefficient and $\Delta r$ is the thickness of the zone considered. It is noticed that at a point far away from the origin, i.e. for a large $r$, the artificial viscosity terms in acceleration equation (2.2) and energy conservation equation (2.3) vanish and the equations reduce to their usual form in slab geometry with modified pressure $(P+Q)$.

In our 1-D spherical simulations, the proposed experiment can be modeled as shown in Figure 2. Assuming perfect spherical symmetry, hydrodynamics inside the dashed sphere is simulated. The total metal thickness should be within the ion range with uniform ion energy deposition, and so $r_{\mathrm{m}}$ is limited in principle. Along the $x$-direction, i.e. along the beam axis, the metal target will expand outward freely to the chamber. But along the $y$-direction, the expansion of the metal outward will be stopped by the target itself. In our 1-D simulations, the outer boundary of the metal is assumed to be either in free expansion or in a fixed position along all directions. The actual situation should be something between these two cases.

In the later part of the present paper we will see that the ion beam leads to rapid collapse of the bubble within about $0.3 \mathrm{~ns}$ and we are mainly interested in the physical environment (e.g., temperature, density and pressure) achievable in that time scale. As the processes of solubilization and evaporation usually takes place in the time scale of microseconds $[26,27]$, we can safely neglect the effects arising from such mechanisms and assume that the total number of gas molecule inside the bubble is constant in the simulations.

\section{EQUATIONS OF STATE}

In our simulations, QEOS (a quotidian equation of state) [28] is used as the equation of state (EOS) for the gas inside the bubble and a Grüneisen-type EOS (GEOS) [29-32] is used for the surrounding metal target.

\section{A. QEOS for the gas bubble}

The QEOS data used in the simulations are calculated by MPQeos code [33]. QEOS is a well-known equation of state model for hydrodynamic simulations of warm dense matter (WDM) and other high-pressure phenomena. It is believed that QEOS is one of the available EOS for material under conditions of temperature and density relevant to the proposed experiment. In QEOS, the electronic properties are obtained from a modified Thomas-Fermi statistical model, while the ion thermal motion is described by a multiphase equation of state combining Debye, Grüneisen, Lindemann, and fluid-scaling laws. QEOS can give smooth predictions for ionization state, pressure, energy, entropy and Helmholtz free energy in hydrodynamic simulations.

\section{B. Grüneisen-type EOS for the metal}

The Grüneisen-type equation of state (GEOS) used is a semi-empirical EOS. The EOS consists of three parts: (i) a zero temperature part which is related to the interaction forces between atoms at zero temperature, (ii) a thermal lattice part which is related to the thermal atomic vibrations about their mean positions, and (iii) an electron thermal part which is related to the thermal excitation of the electrons.

The EOS calculates the Helmholtz free energies per unit mass for solid and liquid seperately. The Helmholtz free energy of the solid phase is given by [30]:

$$
F_{s}(\rho, T)=E_{c}(\rho)+\frac{3 R T}{A} \ln \left[\frac{\Theta(\rho)}{T}\right]-\frac{1}{2} \beta(\rho) T^{2},
$$


where

$$
\begin{aligned}
E_{c}(\rho) & =\frac{3}{\rho_{k}} \sum_{i=1}^{6} \frac{a_{i}}{i}\left[\left(\frac{\rho}{\rho_{k}}\right)^{i / 3}-1\right], \\
\Theta(\rho) & =\rho^{1 / 3}\left(\frac{d P_{c}}{d \rho}-\frac{2}{3} \tilde{t} \frac{P_{c}}{\rho}\right)^{1 / 2}, \\
P_{c}(\rho) & =\rho^{2} \frac{d E_{c}}{d \rho} \\
\beta(\rho) & =\beta_{0}\left(\frac{\rho}{\rho_{k}}\right)^{-1 / 2} .
\end{aligned}
$$

In the above equations $R$ is the gas constant, $A$ is the molar mass of the material, $\rho_{k}$ is the density at $P=0$ and $T=0, E_{c}$ and $P_{c}$ are the internal energy per unit mass and pressure at $T=0$, respectively. In the formula of $F_{s}$, the three separate terms are related to the zero temperature part, the thermal lattice part and the electrons thermal contribution part respectively. $a_{i}, \tilde{t}$ and $\beta_{0}$ are empirical parameters found from experimental data.

The Helmholtz free energy of the liquid phase is given by [32]:

$$
F_{l}(\rho, T)=F_{s}(\rho, T)-\frac{3 R T}{A} \ln \alpha(\rho, T),
$$

where

$$
\begin{aligned}
\alpha(\rho, T) & =(1+z(\rho, T))^{-1 / 2} \exp \left[b-f(\rho) \frac{T_{m 0}}{T}\right], \\
z(\rho, T) & =\frac{l R T}{A}\left(\frac{d P_{c}}{d \rho}-\frac{2}{3} \tilde{t} \frac{P_{c}}{\rho}\right)^{-1}, \\
f(\rho) & =c+\frac{a}{\tilde{r}}\left[\left(\frac{\rho}{\rho_{l 0}}\right)^{\tilde{r}}-1\right],
\end{aligned}
$$

with $T_{m 0}$ and $\rho_{l 0}$ being the melting temperature at $P=0$ and the liquid density at $P=0$ and $T=T_{m 0}$, respectively. In the formula of $F_{l}$, in addition to $F_{s}$, there is one more term related to the phase transition. The phase transition term is an interpolation of pressure and specific heat of the lattice part to their high temperature and low density limit. This term also describes the jumps in entropy and volume during the phase transition. Here, $b, l, c, a$ and $\tilde{r}$ are empirical parameters found from experimental data. The pressure and the internal energy per unit mass of the material can be found by the thermodynamic equalities from the Helmholtz free energy.

The melting curve is the melting temperature as a function of pressure. It can be found by the Clausius-Clapeyron equation [34]:

$$
\frac{d P}{d T}=\frac{L}{T\left(v_{l}-v_{s}\right)},
$$

where $v_{l}=1 / \rho_{l}$ and $v_{s}=1 / \rho_{s} . \rho_{l}$ and $\rho_{s}$ are the liquid and solid density of the material on the melting curve. $L$ is the latent heat of fusion per unit mass and it is given by:

$$
L=H_{l}\left(\rho_{l}, T\right)-H_{s}\left(\rho_{s}, T\right)=E_{l}\left(\rho_{l}, T\right)-E_{s}\left(\rho_{s}, T\right)+P\left(v_{l}-v_{s}\right),
$$

where $H_{l}$ and $H_{s}$ are the enthalpy per unit mass for liquid and solid phases respectively and $E_{l}$ and $E_{s}$ are the internal energy per unit mass for liquid and solid phases respectively. It is noticed that the pressure on the melting curve satisfies:

$$
P=P_{l}\left(\rho_{l}, T\right)=P_{s}\left(\rho_{s}, T\right)
$$

where $P_{l}$ and $P_{s}$ are the pressure for liquid and solid phases respectively. To solve (3.10) for aluminum, the experimental result that the melting temperature of aluminum is $1076 \mathrm{~K}$ at $P=0$ has to be used [35]. The melting curve calculated also fits the experimental data for melting temperature at different pressures [35] and the Gibbs free energies at the liquid and solid phases on the melting curve are also checked to be equal. Within the 2-phase region of solid and liquid (for density between $\rho_{l}$ and $\rho_{s}$ ), the pressure is independent of density and equal to $P$. The internal energy is a linear interpolation of the solid and liquid limits. All the parameters used in the EOS for aluminum are shown in Table I. 


\section{BUBBLE COLLAPSE}

In this section, unless specified, the simulations use argon gas bubbles surrounded by aluminum metal with fixed outer boundaries and experimental parameters of initial gas density of $0.04 \mathrm{~g} / \mathrm{cm}^{3}, r_{\mathrm{b}}$ of $0.5 \mu \mathrm{m}$ and $r_{\mathrm{m}}$ of $2 \mu \mathrm{m}$. The ion beam we consider is the planned NDCX-II experiment at Lawrence Berkeley National Laboratory (LBNL), which has a $\mathrm{Li}$ ion beam with energy of $2.8 \mathrm{MeV}$ and $0.03 \mu \mathrm{C}$ charge in $1 \mathrm{~ns}$ pulse. In this section, the ion energy deposition rate per unit mass is assumed to be constant and uniform inside the target and it is set to be $15 \mathrm{~kJ} / \mathrm{g} / \mathrm{ns}$.

As shown in Figure 3, after the intense iom beam is turned on at $t=0$, ion beam energy will be deposited to the target. The energy input will generate a large pressure difference between the gas bubble and the metal surrounding. The pressure gradient will collapse and heat the gas bubble. After some time, the bubble radius will reach a stagnation point at which the bubble wall velocity turns from negative to positive and rebound (Figure 3a and $\mathrm{b}$ ). At the stagnation point, the hydrodynamic movement of the gas is frozen and the kinetic energy of the gas turns into the internal energy (Figure 3d). At that time, the average temperature and pressure over the whole gas bubble are about $4 \mathrm{eV}$ and $2 \mathrm{Mbar}$ respectively, while the core gas temperature and pressure can go up to $9 \mathrm{eV}$ and 3 Mbar respectively (Figure 3e and f). Most of the energy input to the bubble (more than 90\%) is the work done by the surrounding metal and the direct ion heating just consists of less than $10 \%$ of the total energy gain while the heat conduction effect is also negligible (Figure 3c). Figure $3 \mathrm{~d}$ also shows the values of $3 k T / 2 m$ (the internal translational kinetic energy per unit mass of the gas particles) and compares to the total internal energy. The differences between the two values (about $38 \mathrm{~kJ} / \mathrm{g}$, i.e. $16 \mathrm{eV} /$ ion) are other energy forms such as excitation energy and ionization energy. In Figure 3e and $\mathrm{f}$, the core temperature and pressure curves have sudden jumps at about $0.24 \mathrm{~ns}$, and it is because of the shock waves generated. We will discuss this below (Sec. IV B).

\section{A. First Step of Collapse}

After the target is heated by the ion beam, the pressure of the solid metal, as well as the gas inside the bubble, increases. In a time scale much smaller than the hydrodynamic time, the density can be regarded as constant and so the pressure rise can be estimated by (1.1). Because of the very high bulk modulus of the metal compared to those of the gas, as well as their comparable specific heat capacities, the pressure increase of the metal is much higher than that inside the bubble.

Figure 4 shows the pressure and velocity profiles at different times after the start of the ion beam heating. Just after the target heating starts, there is a large pressure jump (at $t=0.01 \mathrm{~ns}$, the pressure difference is about 6 kbar, while the pressure created by surface tension is about 40 bar) created across the bubble wall. This pressure gradient will push the metal inwards and compress the gas bubble. The compression will heat up the bubble and the kinetic energy of the metal will ultimately become the internal energy of the gas inside the bubble.

\section{B. Stagnation Point and Bubble Size}

After the sharp pressure gradient across the bubble wall is generated when the ion beam is turned on, a spherical shock wave moving inwards to the bubble center will be generated (as shown in Figure 5). Because of the spherical symmetry, the shock wave energy will be more and more concentrated. When the shock wave reaches the center, the gas around the center will be highly compressed and heated and so create sudden jumps in core temperature and pressure (as shown in Figure 3e and f). Then the shock wave will be reflected and propagate outwards. In Figure 5b, the shock wave reaches the center at about $t=0.24 \mathrm{~ns}$ and at $t=0.25 \mathrm{~ns}$, the pressure shock is moving outwards. The outward shock wave will further heat the bubble and so the pressure inside the bubble will be large enough to stop the bubble collapse. In Figure 5b, the outward shock reaches the bubble wall at about 0.26 ns, which is very close to the stagnation time.

Figure 6 shows another case with twice the bubble radius relative to the first simulation and with the same ion energy deposition rate per unit mass. We find again that the shock wave will compress and heat the gas around the bubble center (Figure 6a). Then the shock wave will be reflected (at about $t=0.37$ ns in Figure $6 \mathrm{~b}$ ) and nearly at the time that the reflected shock reaches the bubble wall (at about $t=0.41 \mathrm{~ns}$ ), the collapse stops and rebounds.

\section{Outer Boundary and Metal Thickness}

As discussed before, along the beam axis, the metal target will expand freely to the chamber (as shown in Figure 2 ). At the far end point of the metal target, rarefaction wave will be generated and propagate towards the gas bubble 
when the metal expands. Figure 7 shows the velocity and pressure profiles for a case of free outer boundary. The rarefaction wave generated at the far end point of the target may finally affect the compression of the bubble if the metal thickness $\left(r_{\mathrm{m}}-r_{\mathrm{b}}\right)$ is small enough and there is enough time for it to propagate.

Figure 8 shows the velocity and pressure profiles at a time close to the stagnation time $(t=0.26 \mathrm{~ns})$ for different cases with different outer boundary and $r_{\mathrm{m}}$. For fixed outer boundary cases, there is only one rarefaction wave travelling from the bubble wall to the metal target and the end point. This rarefaction wave will be reflected when it reaches the end point. For free boundary cases, there is another rarefaction wave travelling from the outer boundary end point towards the bubble. If the metal is too thin and/or the stagnation time is too long, this rarefaction wave may reach the bubble before the stagnation point and affect the stagnation pressure. As shown in Figure 8 , the case of $1.5 \mu \mathrm{m} r_{\mathrm{m}}$ with free outer boundary has a much lower stagnation pressure inside the bubble than all other cases. If the metal is thick enough, the rarefaction wave will not be able to affect the bubble and so the bubble will have the same profiles at the stagnation point. In Figure 8, all the other cases have the same pressure and velocity profiles inside the bubble. If it is the case, the bubble will have the same stagnation profiles along both $\mathrm{x}$ and $\mathrm{y}$ directions (as shown in Figure 2) and the bubble will be roughly in spherical symmetry.

As a result, the target and bubble sizes have to be chosen very carefully. (1) The total target thickness $\left(2 r_{\mathrm{m}}\right)$ has to be small enough and within the ion range with uniform energy deposition. (2) The bubble size $\left(r_{\mathrm{b}}\right)$ has to be large enough so that it is easier for diagnostics. (3) The metal thickness $\left(r_{\mathrm{m}}-r_{\mathrm{b}}\right)$ has to be large enough so that the collapse is spherical symmetric. So careful calculations and simulations are essential in order to decide these parameters before the experiment.

\section{Metal Layer Just Outside Bubble}

Just after the bubble starts to collapse, the compression is very fast due to the large pressure difference between the metal and bubble. At this stage, the metal layer around the gas bubble expands inwards just simply because of the thermal expansion. Figure 9a shows the density change of the metal layer just outside the bubble. Before $t=0.21$ $\mathrm{ns}$, that metal layer is still expanding. It can be noticed that at $t=0.05$ to $0.075 \mathrm{~ns}$, that metal layer is in phase transition from solid to liquid and the expansion speed is faster than at other times. During the phase transition, the metal temperature is constant at the melting point of about $1000 \mathrm{~K}$ (as shown in Figure 9c).

As the gas pressure inside the bubble rises, the bubble wall will be decelerated by the gas pressure. But the metal far away from the bubble is still flowing inwards. The large inertia of metal will compress the metal layer around the bubble and create a high density layer of nearly double solid density at about the stagnation time (Figure 9a). As shown in Figure 9b, at stagnation, the metal layer with high density is just about $0.2 \mu \mathrm{m}$ thick and the other part of the metal is still at about the normal density.

At that time, the pressure of the compressed layer is nearly the same as that inside the bubble (2 Mbar, as shown in Figure 9d) but with relatively low temperature of $0.6 \mathrm{eV}$ (Figure 9c).

\section{EFFECT OF EQUATION OF STATE USED}

In the simulations, the results may depend on the equation of state (EOS) used. In the previous sections, we use QEOS as the EOS for the argon gas inside the bubble and the Grüneisen-type equation of state (GEOS) for the aluminum metal surrounding. To test how sensitive the EOS affects the simulation results, we change the EOS.

In this section, the simulations use experimental parameters of ion energy deposition rate per unit mass of 15 $\mathrm{kJ} / \mathrm{g} / \mathrm{ns}$, initial gas density of $0.04 \mathrm{~g} / \mathrm{cm}^{3}, r_{\mathrm{b}}$ of $0.5 \mu \mathrm{m}$ and $r_{\mathrm{m}}$ of $2.5 \mu \mathrm{m}$ with fixed outer boundaries.

\section{A. Inverse Power Potentials Equation of State}

We use an EOS describing inverse power potential between particles and the ionization effect. The intermolecular potentials are assumed to be inversely proportional to the $n$-th power of the separation between two particles. For larger $n$, the EOS is considered to be "harder". This EOS is used for the argon gas inside the bubble and it is denoted 
as inverse power potentials equation of state (IPEOS) [36, 37]. The IPEOS is given by:

$$
\begin{aligned}
& P=\frac{\rho k T}{m}\left(1+Z_{\text {ion }}\right)+\frac{E_{c k} \rho_{k}}{1+(3 / n)}\left[\left(\frac{\rho}{\rho_{k}}\right)^{(n / 3)+1}-\left(\frac{\rho}{\rho_{k}}\right)^{2}\right], \\
& E=\frac{3}{2} \frac{k T}{m}\left(1+Z_{\text {ion }}\right)+\frac{Q\left(Z_{\text {ion }}\right)}{m}+\frac{E_{c k}}{(3 / n)-1}\left[\left(\frac{\rho}{\rho_{k}}\right)^{(n / 3)}-\frac{n}{3}\left(\frac{\rho}{\rho_{k}}\right)\right],
\end{aligned}
$$

where $m, k, \rho_{k}$ and $E_{c k}$ are the mass of a single gas atom, the Boltzmann constant, the density of the solid gas at $T=0$ (for argon gas, $\rho_{k}=1.626 \mathrm{~g} / \mathrm{cm}^{3}$ ) and the binding energy per unit mass of solid gas at $T=0$ (for argon, $\left.E_{c k}=195 \mathrm{~J} / \mathrm{g}\right) . Z_{\text {ion }}$ is the charge state of the gas, i.e. the total number of free electrons divided by the total number of gas atom and ion, and $Q\left(Z_{\text {ion }}\right)$ is the total energy needed to remove $Z_{\text {ion }}$ electrons from an atom. In $(5.1)$ and (5.2), the last term is a simplification of the results of Monte Carlo calculations of intermolecular potentials in fluids and solids [37].

To calculate $Z_{\text {ion, }}$, we use an implicit equation derived from the Saha equation for the average degree of ionization [38]:

$$
I\left(Z_{\text {ion }}+0.5\right)=k T \ln \left(\frac{0.01 A[k T(\mathrm{eV})]^{3 / 2}}{Z_{\mathrm{ion}} \rho\left(\mathrm{g} / \mathrm{cm}^{3}\right)}\right),
$$

where $I\left(Z_{\text {ion }}\right)$ is the ionization potential for any degree of ionization $Z_{\text {ion }}$. For integer $Z_{\text {ion }}, I\left(Z_{\text {ion }}\right)$ can be found from literatures [39] and $Q\left(Z_{\text {ion }}\right)=\sum_{m=1}^{Z_{\text {ion }}} I(m)$. For non-integer $Z_{\text {ion }}, I\left(Z_{\text {ion }}\right)$ and $Q\left(Z_{\text {ion }}\right)$ can be found by interpolation.

Figure 10 shows the simulation results using IPEOS with different $n$ and QEOS for the argon gas inside the bubble. For a "softer" IPEOS (i.e. with a smaller $n$ ), the bubble will compress more (Figure 10a) and result in a higher temperature (Figure 10c). In general, using IPEOS will have higher stagnation temperature and pressure than using QEOS, even though their stagnation radii are nearly the same. A possible reason for the difference is that QEOS uses Thomas-Fermi (TF) model to calculate the ionization while IPEOS uses Saha model and TF model usually gets a higher ionization state than the Saha model (for the case here, the maximum ionization state for IPEOS is about 0.5 while that for QEOS is 3.5, while at $t=0$, IPEOS gets zero charge state and QEOS gets 0.3 charge state). So much more energy is used for ionization for QEOS and the stagnation temperature, and so pressure, will be lower.

\section{B. Effect of Ionization}

To test the effect of ionization in the simulaitons, IPEOS is used with $Z_{\text {ion }}$ set to be zero. The results are then compared to that for the original IPEOS. The results for $n=12$ are shown in Figure 11 . It is noticed that the case without ionization will have stagnation temperature of $15.5 \mathrm{eV}$ while that for the case with ionization is $6.5 \mathrm{eV}$ (Figure 11c). The charge state for case with ionization is about 0.5 and the first ionization energy of argon is 15.8 $\mathrm{eV}$. So the difference between the stagnation temperatures of the two cases is mainly due to the ionization energy of the gas. The case without ionization will have a larger stagnation bubble radius and so the stagnation pressure will be smaller.

\section{Effect of Hard Core}

Beside IPEOS, we also use Van der Waals' EOS (VDW-EOS) for the gas in the simulations. The VDW-EOS is given by:

$$
\begin{aligned}
P & =\frac{\rho k T}{m(1-\tilde{b} \rho)}-\tilde{a} \rho^{2} \\
E & =\frac{3}{2} \frac{k T}{m}-\tilde{a} \rho \\
\tilde{b} & =\frac{1+\sqrt{1-\left(32 T_{r}\right) /\left(27 T_{c}\right)}}{2 \rho_{k}} \\
\tilde{a} & =\frac{27}{8} \frac{k T_{c}}{m} \tilde{b}
\end{aligned}
$$

where $T_{c}$ is the critical temperature (for argon gas, $T_{c}=0.013 \mathrm{eV}$ ). Instead of the usual formula of $\tilde{b}=1 / \rho_{k}$, a small thermal expansion effect is added $[23,40]$ and $(5.6)$ is used to calculate $\tilde{b}$. $T_{r}$ is a reference temperature so that at 
$T=T_{r}$ and $\rho=\rho_{k}, P=0$. Otherwise, an infinite pressure will be obtained at solid density. For argon gas, $T_{r}$ is set to be the melting temperature at $P=0(=0.0072 \mathrm{eV})$. The VDW-EOS is also modified by the Maxwell construction so as to describe the liquid-vapor two-phase region. The VDW-EOS can be treated as a "completely hard" EOS in the sense of IPEOS. We use a VDW-EOS with no ionization and so we may compare the results to IPEOS without ionization $(n=12)$, as shown in Figure 12. In (5.4), because of the denominator of $(1-\tilde{b} \rho)$, the density is limited by $1 / \tilde{b}$. So the compression ratio for the case of VDW-EOS will be limited and smaller than using IPEOS (Figure 12a). The weaker compression for VDW-EOS will result in lower stagnation temperature and pressure (Figure 12c and d).

\section{Effect of EOS for metal}

We also investigate the effect of the EOS used for the metal on the bubble collapse. We compare the results of using VDW-EOS for the metal to the results using GEOS.

For the case of using VDW-EOS for the metal, (5.4), (5.5), (5.6) and (5.7) are used to find the pressure, internal energy per unit mass, $\tilde{b}$ and $\tilde{a}$ of the metal respectively. Under room conditions, the metal density is very close to $\rho_{k}$ (the solid density at $T=0$ ) because of the low thermal expansion coefficient. So $T_{r}$ is set to be the room temperature $(=0.025 \mathrm{eV})$ and (5.4) will give a finite pressure equal to 0 under room temperature and solid density.

For both cases, we use QEOS as the gas EOS in the simulations and the results are shown in Figure 13. As shown in Figure 13a, the compression for the case of VDW-EOS is much faster than that for the case of GEOS. The much faster compression will result in a shorter stagnation time, smaller stagnation radius and much higher stagnation temperature and pressure (Figure 13a, c and d).

To explain the very fast compression, we may have to find the $(\partial P / \partial E)_{V}$ values for the two EOS from (1.1) under normal conditions. Table II shows the coefficients of thermal expansion, the bulk modului, the specific heat capacities and $(\partial P / \partial E)_{V}$ values for VDW-EOS, GEOS and the experimental values. We find that these values for GEOS are closer to the experimental values. VDW-EOS has a too small thermal expansion coefficient and specific heat capacity (about half the experimental values). But it has a much higher bulk modulus (more than 50 times higher). As a result, VDW-EOS will get a very high $(\partial P / \partial E)_{V}$ value (about 40 times higher). So for the same energy deposition, the pressure gradient generated for the VDW-EOS case will be much higher than that for GEOS and so cause a much stronger compression.

We conclude that the EOS used for metal will affect the bubble collapse significantly and so the proposed experiment is likely to examine the EOS for the metal as well as that for the gas.

\section{EFFECT OF EXPERIMENTAL PARAMETERS}

\section{A. Initial Gas Density}

In the experiment, a gas bubble is created inside a solid metal target initially. After creating the bubble, the gas pressure inside bubble is the same as that of the solid metal with the surface tension correction by (2.1). Using different methods to create the bubble, the initial gas pressure and so the gas density can be different. So the initial gas density can be treated as an experimental parameter in the simulation. After we make a small increase in the gas density, the gas pressure increases and so we have to increase the metal density (and so the pressure) so as to equate the gas and metal pressure (with surface tension correction). Because the metal has high bulk modulus, the change in metal density will be very small.

Figure 14 shows the simulation results for different initial gas densities. For the case of higher initial gas density, more gas is inside the bubble. So it is harder to compress the bubble to a small size and the collapse is weaker. As a result, the stagnation radius will be larger (Figure 14a). For the lower density case, because of the stronger compression, the stagnation temperature and pressure will be higher (Figure 14c to f). Figure 14b shows the pressure profiles at stagnation point for the cases. The differences in pressure and stagnation radius are indicated.

\section{B. Energy Deposition Rate}

In the experiment, the ion energy deposition rate to the target depends on the kind of ion used in the beam, the ion beam energy, the beam charge density, the target material, etc. Because we assume that the ion energy deposition rate is constant and uniform, it can be treated as one of the experimental parameters that can be changed. For lower energy deposition rate, the increase in metal pressure is lower and so the collapse is slower, and it results in lower 
stagnation temperature and pressure. Figure 15 shows the simulation results for different ion energy deposition rates. For the case of lower deposition rate, the compression rate will be lower and so the stagnation time is larger, while the stagnation radius is close to other cases (Figure 15a). The slower compression also results in lower stagnation temperature and pressure (Figure 15c to f). Figure 15b shows the pressure profiles at stagnation point for the cases. The differences in pressure and stagnation radius are indicated.

\section{CONCLUSION AND DISCUSSION}

In summary, a new experiment is proposed in this paper to produce matter within the HEDP regime in the laboratory. The idea is to use an intense heavy ion beam to heat a solid metal target containing a spherical gas bubble. A large pressure difference between the metal and the gas will be generated within a short time, creating a strong collapse of the gas bubble. At the stagnation of the compression, the maximum core (average) temperature and pressure inside the bubble can go up to $9 \mathrm{eV}(4 \mathrm{eV})$ and $3 \mathrm{Mbar}$ (2 Mbar) respectively, which are both in the HEDP regime. Thus, our proposal here is likely to be a novel idea to attain a few Mbar pressure in the laboratory using intense heavy ion beams. The ultimate goal is to be able to perform experiments on matter in the Warm Dense Matter Regime, an area which is the subject of intense interest. For example, one class of experiments will be to refine equations of state in this regime of high pressure and relatively low temperatures for both gases and metals.

This paper has focused on the creation of matter in the HEDP regime. Diagnosing such a target will be extremely complicated and more work is required to evaluate possible methods for diagnosing the properties of the heated material. We would suggest that the first area to look is for radiation output. This is a non-intrusive diagnostic, and in general could yield detailed information on spectrum, pulse shape, etc. A thorough search for a solid material that will permit transmission over some part of the spectrum would have to be carried out. The optimal size of the bubble and collapse time can be determined based on the beam characteristics (pulse length, deposition intensity, etc.) of the particular ion-beam facility where the experiment is to be performed. It might also be possible to seed the bubble and/or the target with a material that has a characteristic spectra that radiates under known conditions. These diagnostic possibilities require further evaluation based on choices of specific ion beams and target materials.

In our numerical simulations we have neglected effects due to asymmetry in the driving pressure, in the following we show that such approximation is reasonable and would not give rise to serious errors. For concreteness, we consider the NDCX-II beam, which will have an approximately gaussian radial profile with a $0.5 \mathrm{~mm}$ rms radius. Assuming a gaussian profile, there will be an approximate transverse non-uniformity of $\exp \left[-(0.5 / 500)^{2}\right]$ variation over the $0.5 \mu \mathrm{m}$ radius bubble, which is a variation of only $10^{-6}$ from center to edge of the bubble. This radial variation will be swamped by the longitudinal variation arising from the energy change of the ion beam as it slows down through the target. By choosing the energy of the ion such that its Bragg peak (i.e. peak in $d E / d X$ as a function of energy) is near the center of the bubble, we attempt to minimize this energy variation. Nevertheless, over the one-micron diameter of the bubble in the solid, $d E / d X$ will vary by approximately $0.3 \%$, corresponding to a similar variation in temperature and pressure. Since $r_{0} \sim a t_{0}^{2} / 2$, where $a$ is the pressure induced acceleration, $r_{0}$ is the original bubble radius and $t_{0}$ is the bubble collapse time, this indicates $\delta r / r_{0} \sim 0.3 \%$, where $\delta r$ is the lag in radius of the low pressure part of the bubble relative to the high pressure part. This will correspond of a variation in radius of $\sim 0.0015 \mu \mathrm{m}$. The nominal minimum radius from the simulations is $\sim 0.1 \mu \mathrm{m}$ so the $0.3 \%$ energy deposition variation, gets converted to $\mathrm{a} \sim 1.5 \%$ variation in the radius of the ellipsoid at maximum compression. This would lead to errors in deduced maximum pressure of a similar order, which is still a relatively small error compared to present measurements of EOS in this regime. Two-dimensional simulations using multi-dimensional codes (such as Hydra) will be used to validate these estimates.

As we have noted in the Introduction, the present work was originally motivated by related studies in SBSL. Just as SBSL has opened up a completely new field of study with many interesting and new phenomena previously unforeseen, we expect that the proposed experiments with ion beams, if implemented, will lead similarly to a rich new field.

\section{Acknowledgments}

We thank B. G. Logan for proposing the original idea of the collapsing bubble, and A. Friedman, E. Henestroza, B. G. Logan, R. M. More and J. S. Wurtele for discussions. We also thank R. M. More for providing DISH code for hydrodynamic simulations, and A. J. Kemp and J. Meyer-ter-Vehn for providing MPQeos code for calculating QEOS

This work performed under the auspices of the U.S. Department of Energy by Lawrence Livermore National Laboratory under Contract DE-AC52-07NA27344. 
data.

[1] R. C. Davidson et al., Frontiers in High Energy Density Physics: The X-Games of Contemporary Science (The National Academies Press, Washington, D.C., 2003).

[2] F. M. Bieniosek et al., Nucl. Instr. and Meth. A 577, 284 (2007).

[3] J. J. Barnard et al., Nucl. Instr. and Meth. A 577, 275 (2007).

[4] L. R. Grisham, Phys. Plasmas 11, 5727 (2004).

[5] D. H. H. Hoffmann et al., Laser and Particle Beams 23, 47 (2005).

[6] W. L. Waldron et al., Plans for warm dense matter experiments and IFE target experiments on NDCX-II, Conference paper: TOFE 2008 (2008).

[7] N. A. Tahir et al., Nucl. Instr. and Meth. A 544, 16 (2005).

[8] N.A. Tahir et al., Phys. Rev. Lett. 95, 035001 (2005).

[9] N.A. Tahir et al., Phys. Rev. E 63, 016402 (2001).

[10] N.A. Tahir et al., New J. Phys. 12, 073022 (2010).

[11] Toru Sasaki, et al., Nucl. Instr. and Meth. A 606, 161 (2009).

[12] An. Tauschwitz, et al., HEDP 3, 371(2007)

[13] J. D. Lindl, Inertial Confinement Fusion, The Quest for Ignition and Energy Gain Using Indirect Drive (Springer: New York, N.Y., 1998).

[14] S. Atzeni and Jurgen Meyer-ter-Vehn, The physics of inertial fusion (Clarendon Press, Oxford, 2004).

[15] B. Yu. Sharkov et al., Nucl. Instr. and Meth. A 415 (1998) 20-26."

[16] B. P. Barber, R. A. Hiller, R. Lofstedt, S. J. Puttermsn, and K. R. Weninger, Phys. Rep. 281, 65 (1997).

[17] D. Hammer and L. Frommhold, J. Mod. Opt. 48, 239 (2001).

[18] M. P. Brenner, S. Hilgenfeldt, and D. Lohse, Rev. Mod. Phys. 74, 425 (2002).

[19] S. Hilgenfeldt, S. Grossmann, and D. Lohse, Phys. Fluids 11, 1318 (1999).

[20] C. Y. Ho, L. Yuan, M. C. Chu, P. T. Leung, and W. Wei, Phys. Rev. E 65, 041201 (2002).

[21] W. K. Tse and P. T. Leung, Phys. Rev. E 73, 056302 (2006).

[22] S. F. Ng, J. J. Barnard, P. T. Leung, and S. S. Yu, Nucl. Instr. and Meth. A 606, 172 (2009).

[23] R. M. More, DISH User Manual (2007).

[24] J. Armijo, J. J. Barnard, and R. M. More, Poster in American Physical Society, Division of Plasma Physics Meeting (2006).

[25] W. F. Noh, J. Comput. Phys. 72, 78 (1987).

[26] B. D. Storey and A. J. Szeri, Proc. R. Soc. London, Ser. A 456, 1685 (2000).

[27] K. Yasui, Phys. Rev. E 56, 67506760 (1997).

[28] R. M. More, K. H. Warren, D. A. Young, and G. B. Zimmerman, Phys. Fluids 31, 3059 (1988).

[29] L. V. Al'tshuler, S. B. Kormer, A. A. Bakanova, and R. F. Trynin, Sov. Phys. JEPT 11, 573 (1960).

[30] S. Eliezer, A. Ghatak, and H. Hora, An Introduction to Equation of State: Theory and Applications (Cambridge University Press, 1986).

[31] S. B. Kormer and V. D. Urlin, Sov. Phys. Doklady 5, 317 (1960).

[32] V. D. Urlin, Sov. Phys. JEPT 22, 341 (1966).

[33] A. Kemp and J. Meyer-ter Vehn, Nucl. Instr. and Meth. A 415, 674 (1998).

[34] M. W. Zemansky and R. H. Dittman, Heat and Thermodynamics (McGraw-Hill, 1997).

[35] Y. Zou and L. Chen, Phys. Stat. Sol. (b) 242, 2412 (2005).

[36] W. C. Moss, D. B. Clarke, J. W. White, and D. A. Young, Phys. Fluids 6, 2979 (1994).

[37] W. G. Hoover, S. G. Gray, and K. W. Johnson, J. Chem. Phys. 55, 1128 (1971).

[38] R. J. Harrach and F. J. Rogers, J. Appl. Phys. 52, 5592 (1981).

[39] NIST Atomic Spectra Database: http://physics.nist.gov/PhysRefData/ASD/index.html.

[40] R. M. More, DISH code, 2007. 
TABLE I: Parameters of the Grüneisen-type equation of state (GEOS) for aluminum [31, 32]

\begin{tabular}{|l|r||c|c|}
\hline$a_{1}$ & $-1.9228 \mathrm{Mbar}$ & $A$ & $26.98 \mathrm{~g} / \mathrm{mol}$ \\
\hline$a_{2}$ & $18.2301 \mathrm{Mbar}$ & $\tilde{t}$ & 2 \\
\hline$a_{3}$ & $-49.2719 \mathrm{Mbar}$ & $l$ & 6 \\
\hline$a_{4}$ & $53.4701 \mathrm{Mbar}$ & $\tilde{r}$ & 1 \\
\hline$a_{5}$ & $-24.7348 \mathrm{Mbar}$ & $a$ & 1.28 \\
\hline$a_{6}$ & $4.2293 \mathrm{Mbar}$ & $b$ & 0.386 \\
\hline$\rho_{k}$ & $2.745 \mathrm{~g} / \mathrm{cm}^{3}$ & $c$ & 0.308 \\
\hline$\beta_{0}$ & $5.18 \times 10^{-5} \mathrm{~J} / \mathrm{g} \mathrm{K}^{2}$ & $T_{m 0}$ & $1076 \mathrm{~K}$ \\
\hline$R$ & $8.31451 \mathrm{~J} / \mathrm{K} / \mathrm{mol}$ & $\rho_{l 0}$ & $2.28651 \mathrm{~g} / \mathrm{cm}^{3}$ \\
\hline
\end{tabular}

TABLE II: The coefficients of thermal expansion, the bulk modului, the specific heat capacities and $(\partial P / \partial E)_{V}$ values for different EOS under normal conditions.

\begin{tabular}{|l|c|c|c|c|}
\hline & $\alpha\left(\times 10^{-5} \mathrm{~K}^{-1}\right)$ & $K(\mathrm{Mbar})$ & $c_{v}(\mathrm{~J} / \mathrm{g} / \mathrm{K})$ & $(\partial P / \partial E)_{V}(\mathrm{~g}$ bar $/ \mathrm{J})$ \\
\hline experimental values & 6.9 & 0.76 & 0.90 & 58.3 \\
\hline GEOS & 5.0 & 0.71 & 0.94 & 37.8 \\
\hline VDW-EOS & 2.6 & 42.50 & 0.46 & 2400 \\
\hline
\end{tabular}

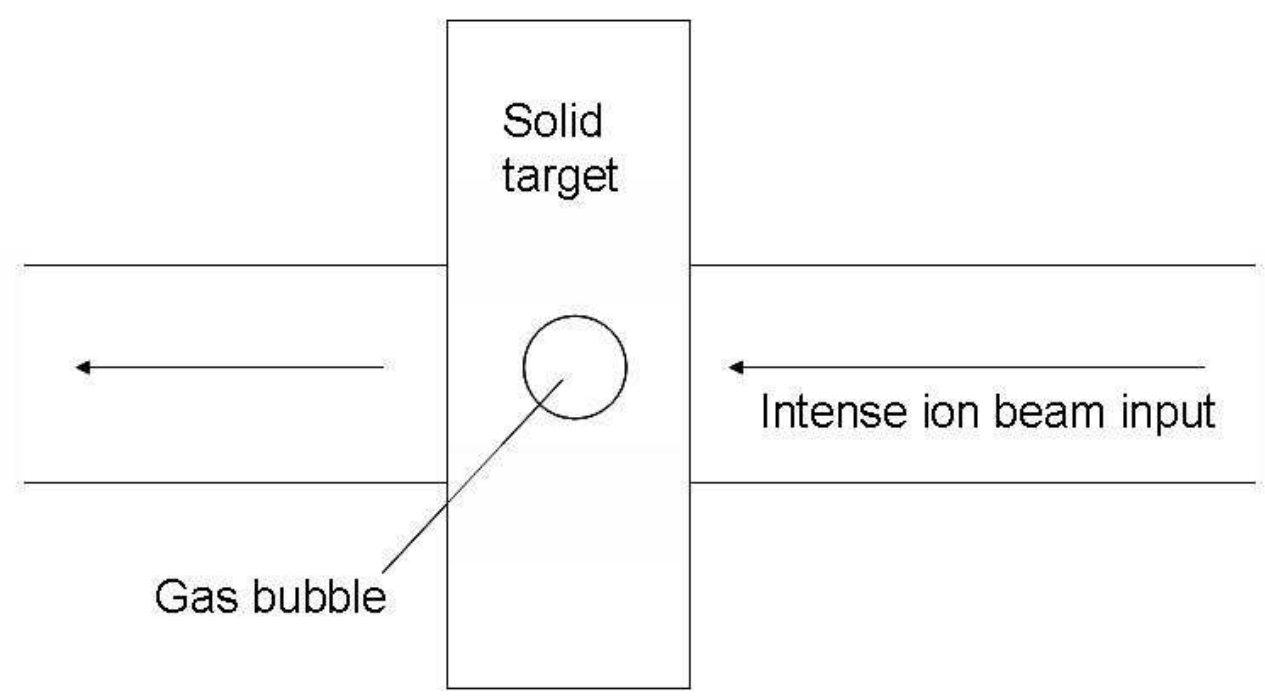

FIG. 1: The proposed experimental setup of a collapsing bubble in a solid metal driven by an intense ion beam. 


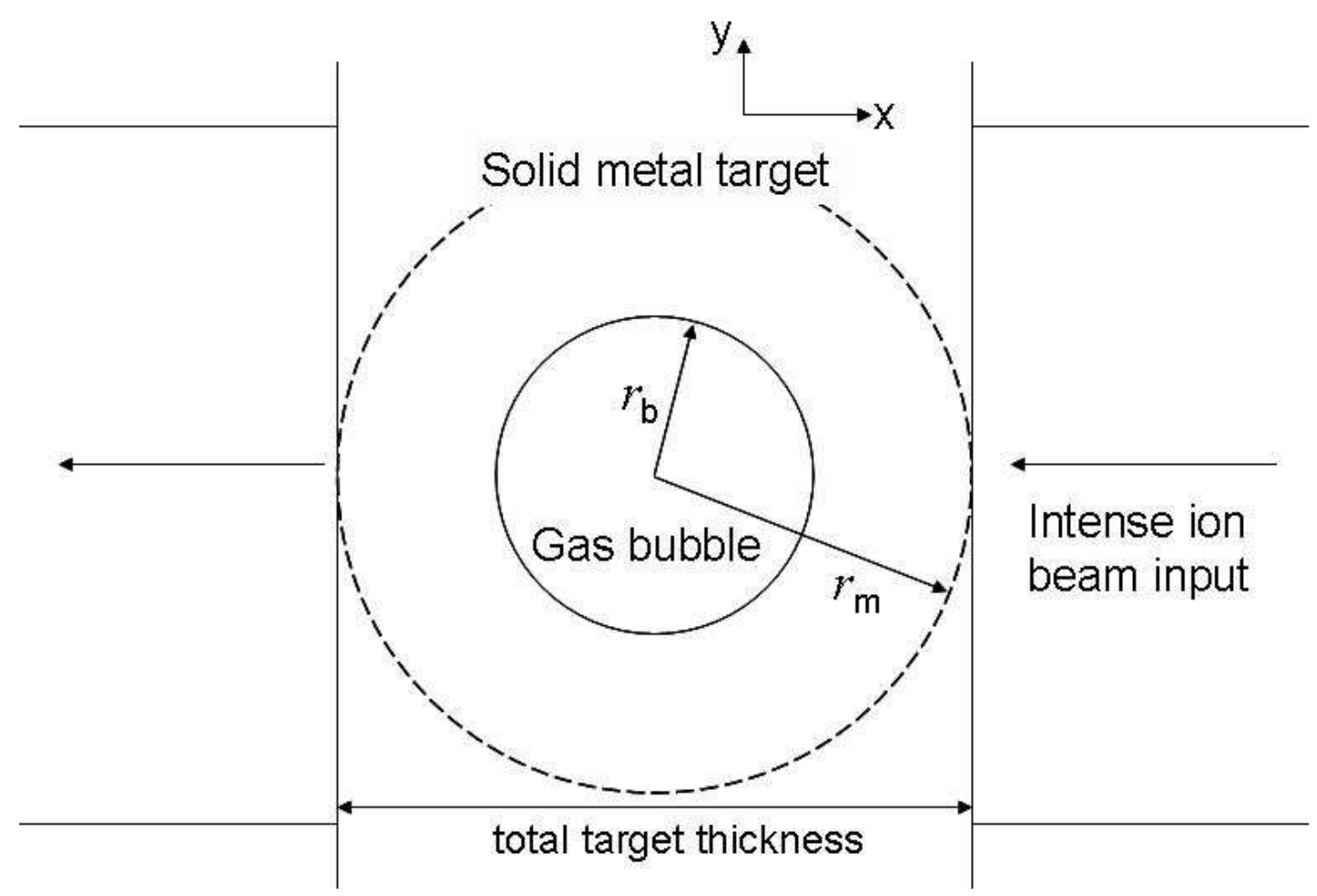

FIG. 2: The geometry of the model used in numerical simulations. 


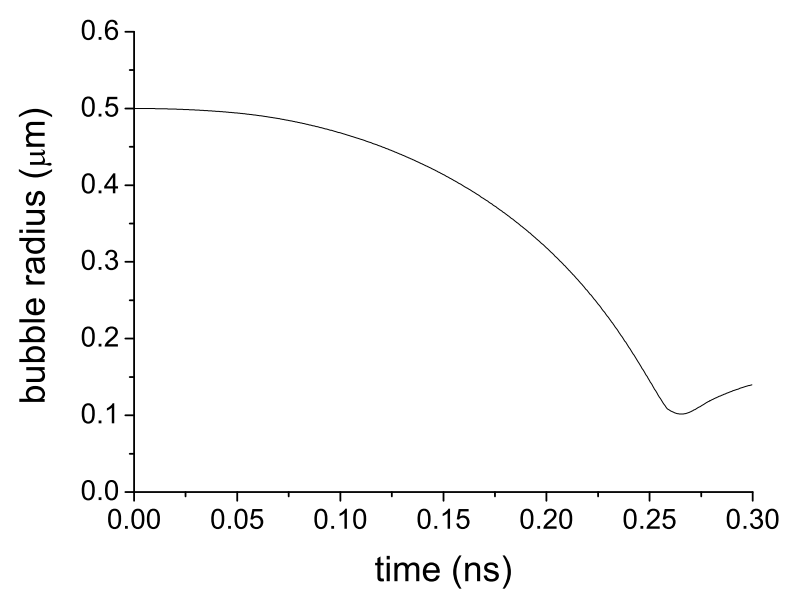

(a)

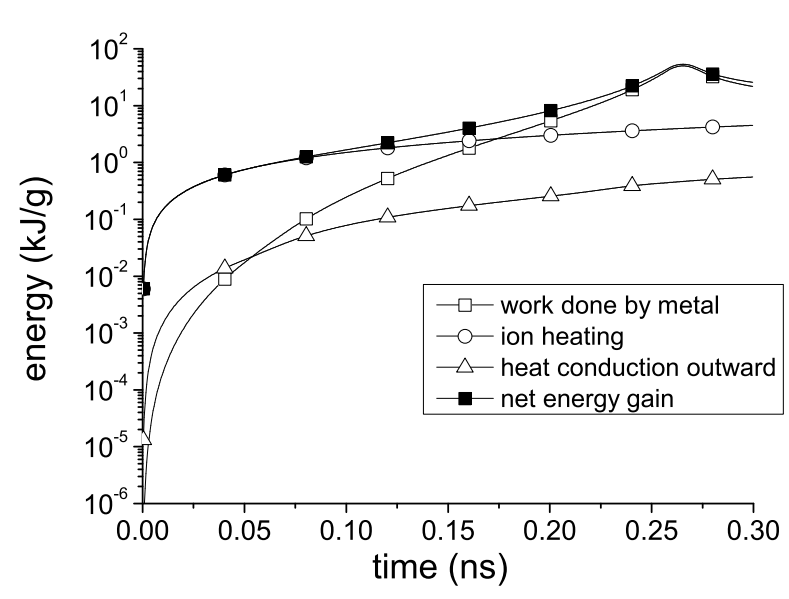

(c)

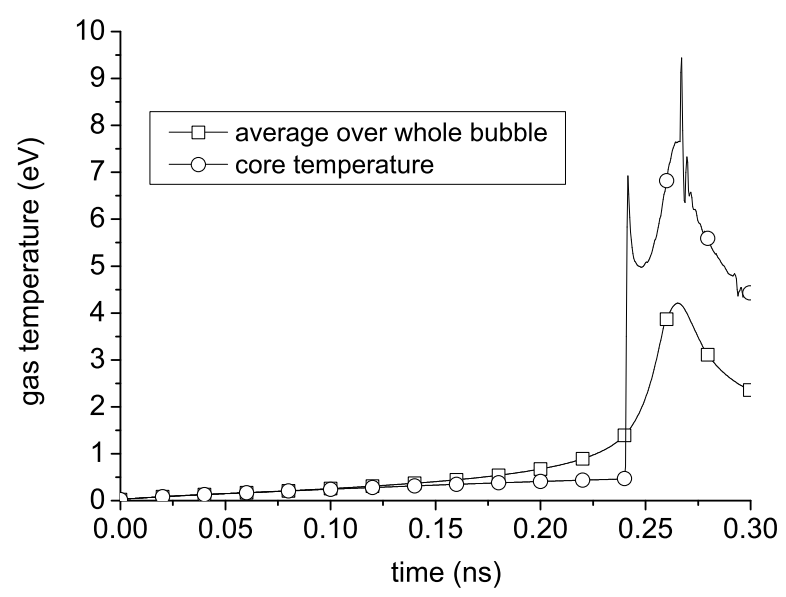

(e)

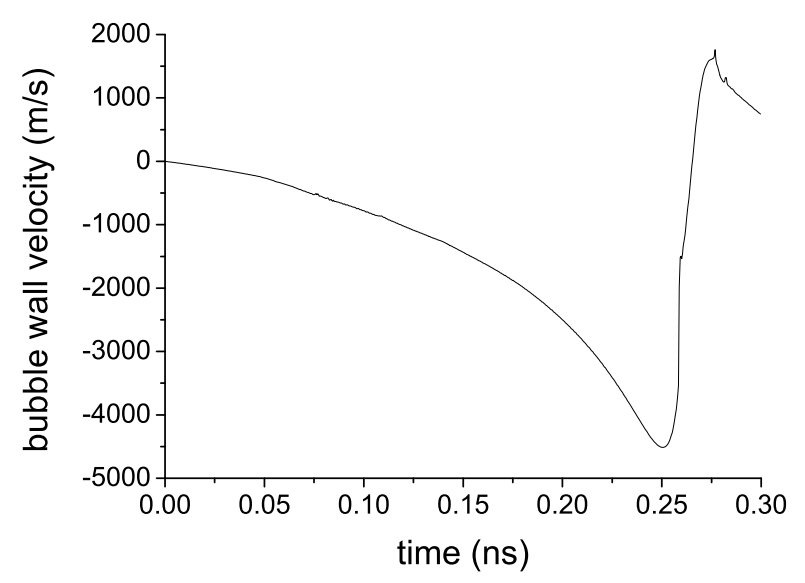

(b)

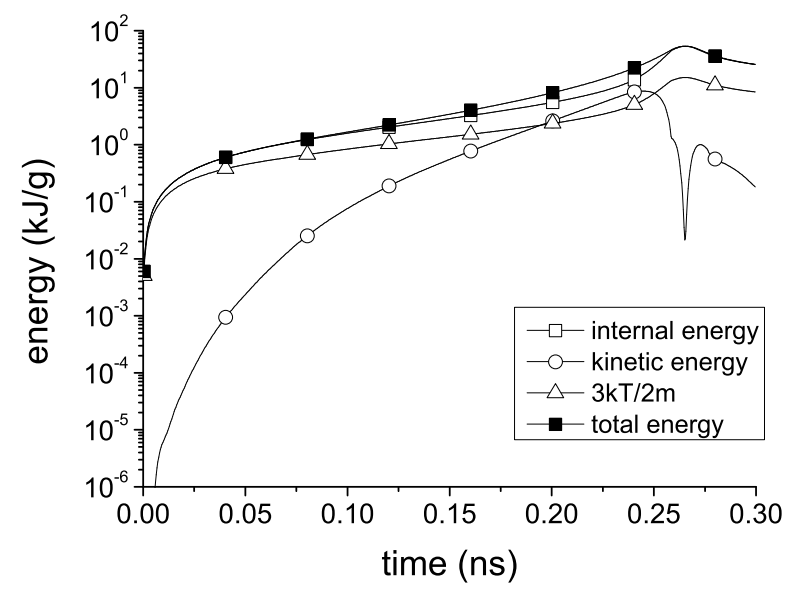

(d)

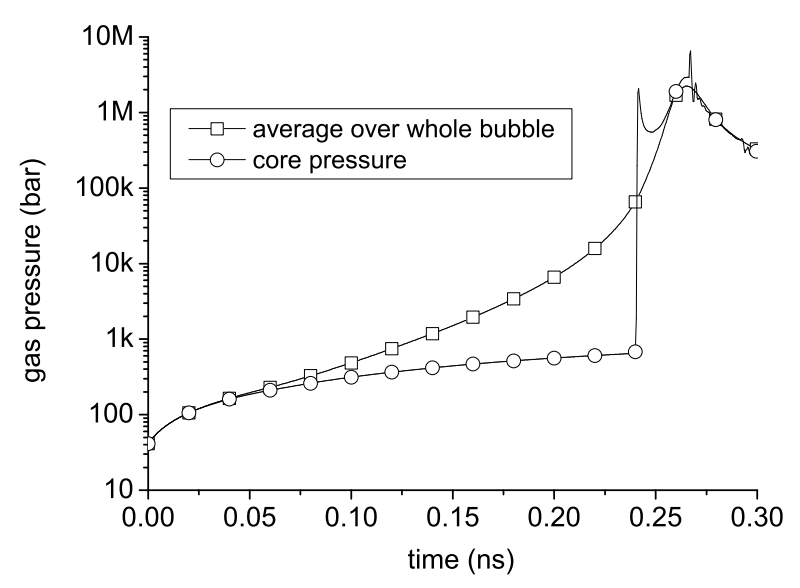

(f)

FIG. 3: (a) Bubble radius, (b) bubble wall velocity, (c) energy input, (d) energy forms inside bubble, (e) gas temperature and (f) gas pressure inside the bubble as functions of time after the ion beam is turned on. 


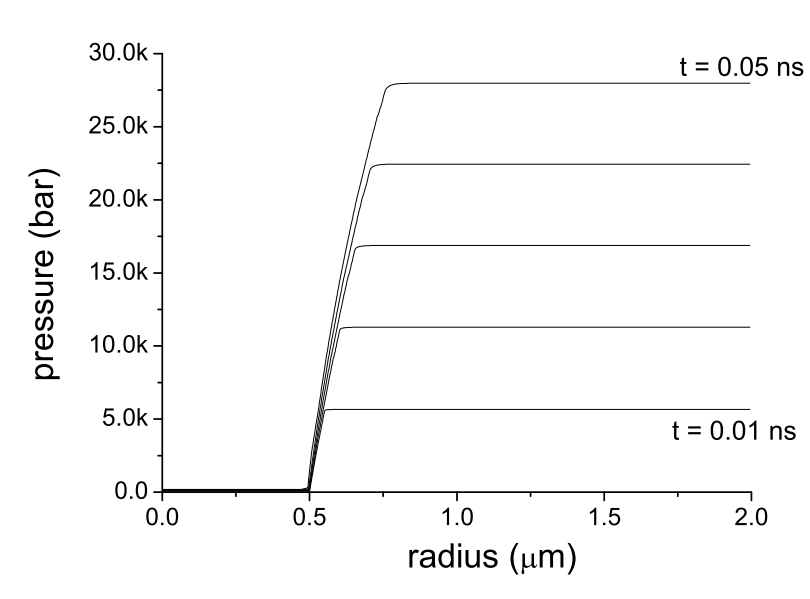

(a)

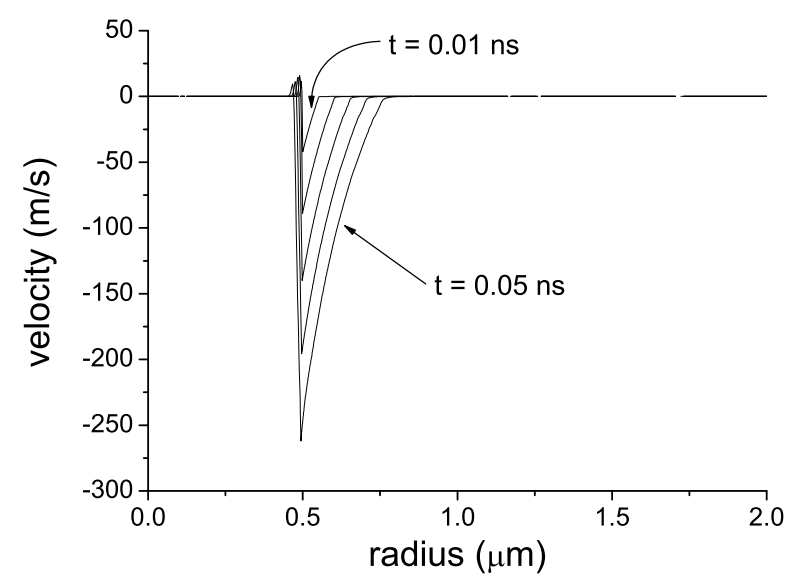

(b)

FIG. 4: (a) Pressure and (b) velocity profiles at $t=0.01,0.02,0.03,0.04,0.05 \mathrm{~ns}$. The bubble radii are about $0.5 \mu \mathrm{m}$ at these times.

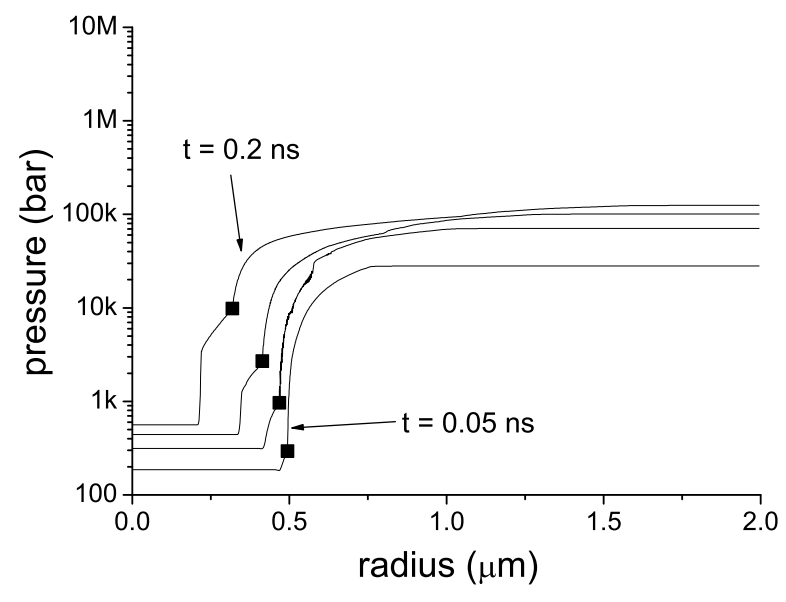

(a)

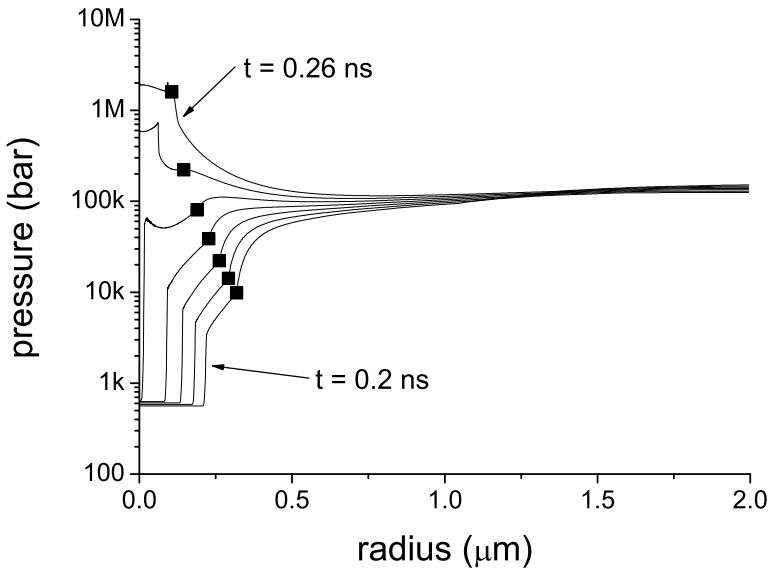

(b)

FIG. 5: Pressure profiles for the case of $r_{\mathrm{b}}=0.5 \mu \mathrm{m}$ and $r_{\mathrm{m}}=2 \mu \mathrm{m}$ at (a) $t=0.05,0.1,0.15,0.2 \mathrm{~ns}$ and (b) $t=0.2,0.21,0.22,0.23,0.24,0.25,0.26 \mathrm{~ns}$. The solid squares indicate the positions of the bubble wall at different times. 


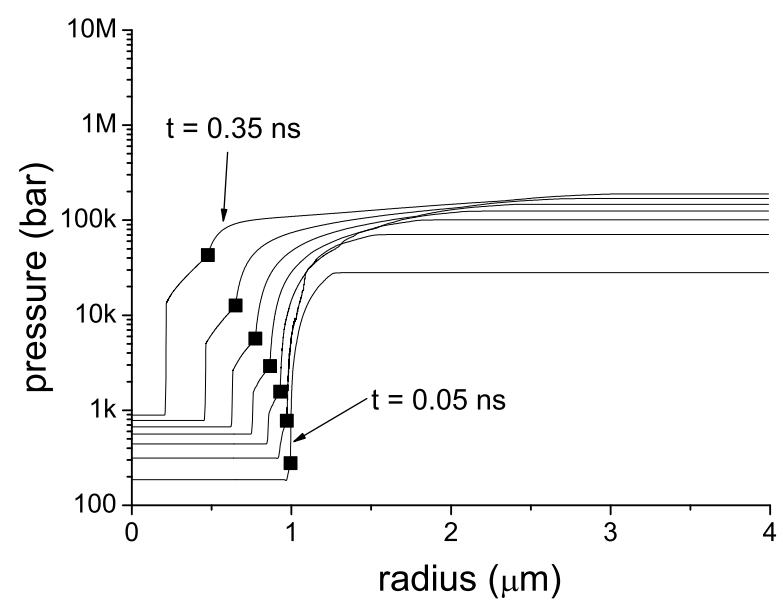

(a)

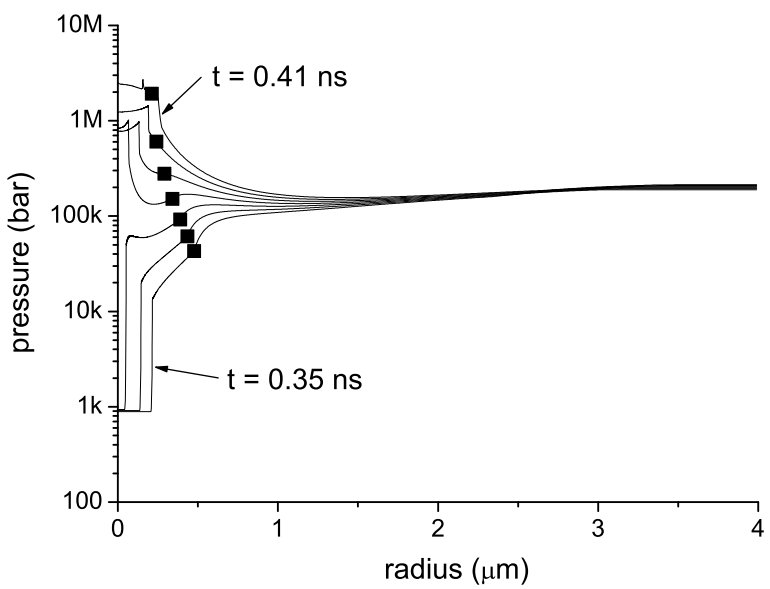

(b)

FIG. 6: Pressure profiles for the case of $r_{\mathrm{b}}=1 \mu \mathrm{m}$ and $r_{\mathrm{m}}=4 \mu \mathrm{m}$ at (a) $t=0.05,0.1,0.15,0.2,0.25,0.3,0.35 \mathrm{~ns}$ and (b) $t=0.35,0.36,0.37,0.38,0.39,0.4,0.41 \mathrm{~ns}$. The solid squares indicate the positions of the bubble wall at different times.

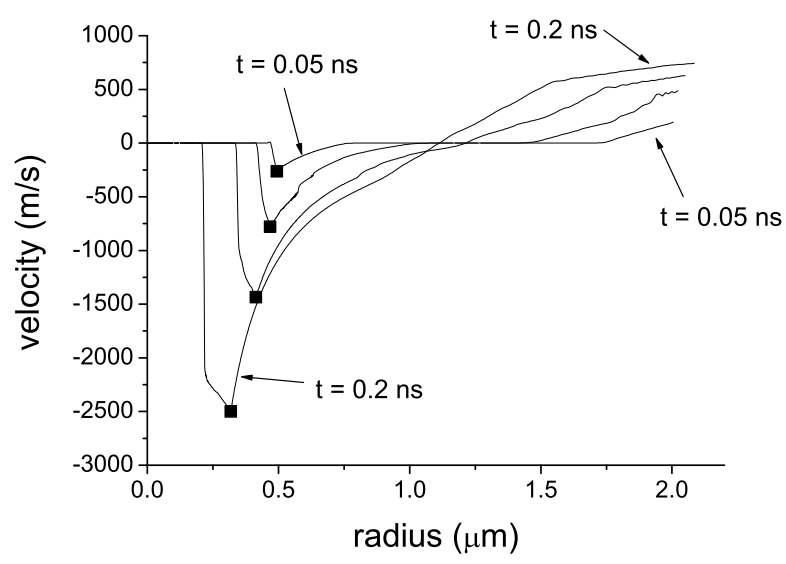

(a)

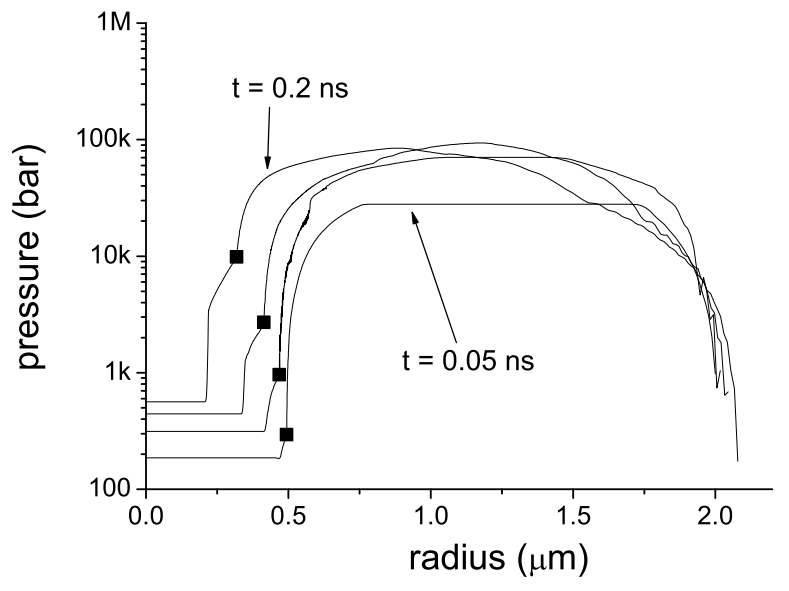

(b)

FIG. 7: (a) Velocity and (b) pressure profiles at $t=0.05,0.1,0.15,0.2 \mathrm{~ns}$ for the case with free outer boundary. 


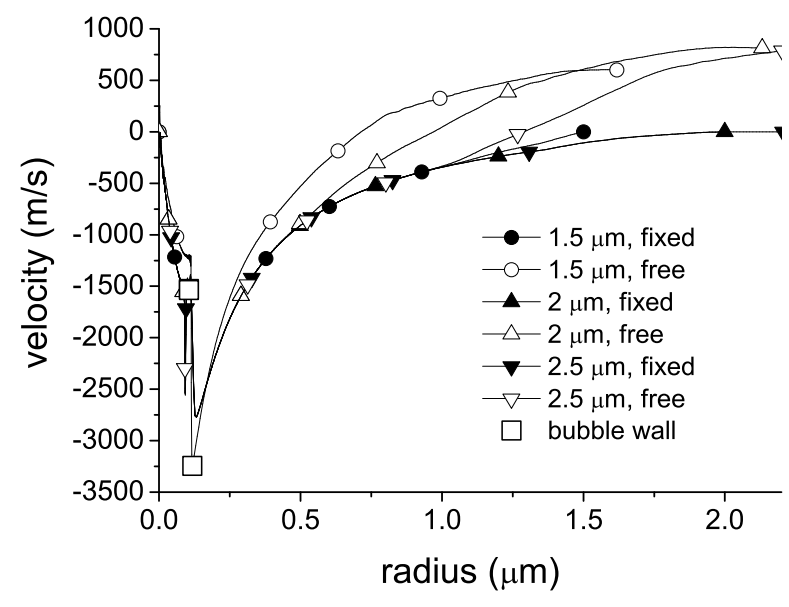

(a)

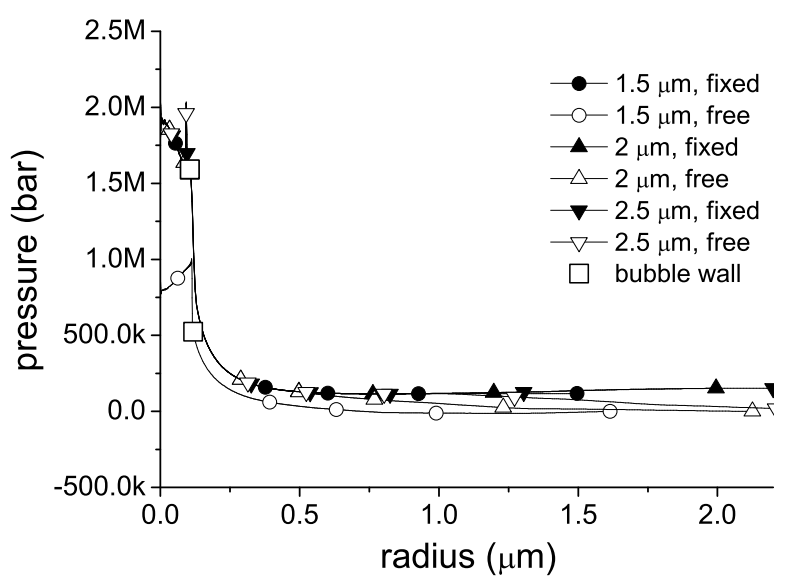

(b)

FIG. 8: (a) Velocity and (b) pressure profiles at $t=0.26 \mathrm{~ns}$ for cases with fixed (or free) outer boundary and different values of $r_{\mathrm{m}}$ as shown in the legends. Except for the case with $r_{\mathrm{m}}=1.5 \mu \mathrm{m}$ and free outer boundary, all cases shown here have the same profile around the gas bubble. 


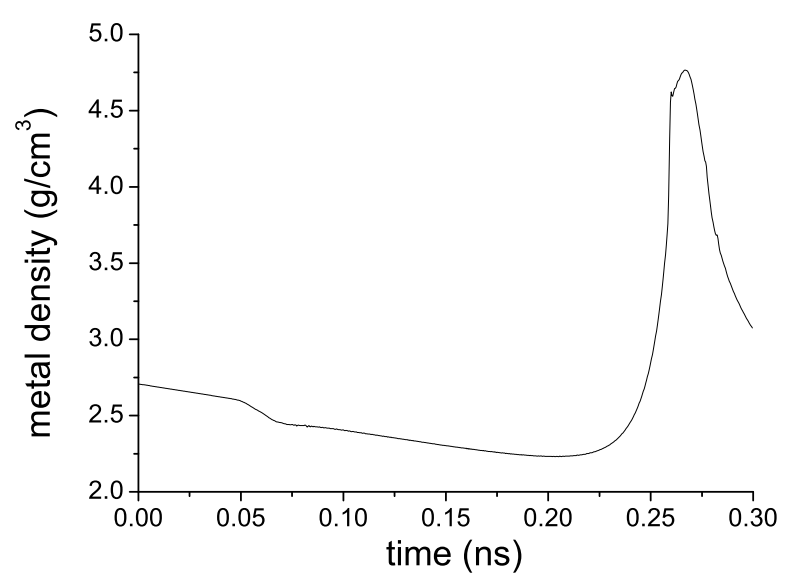

(a)

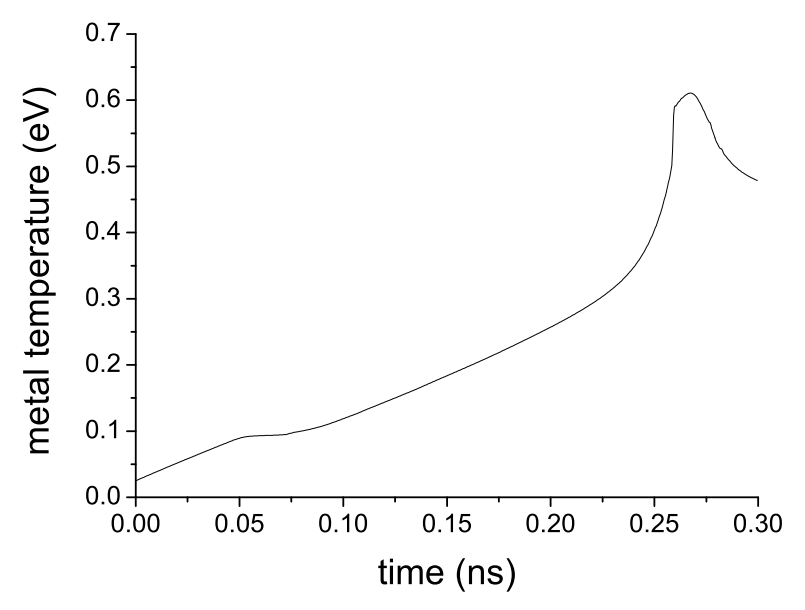

(c)

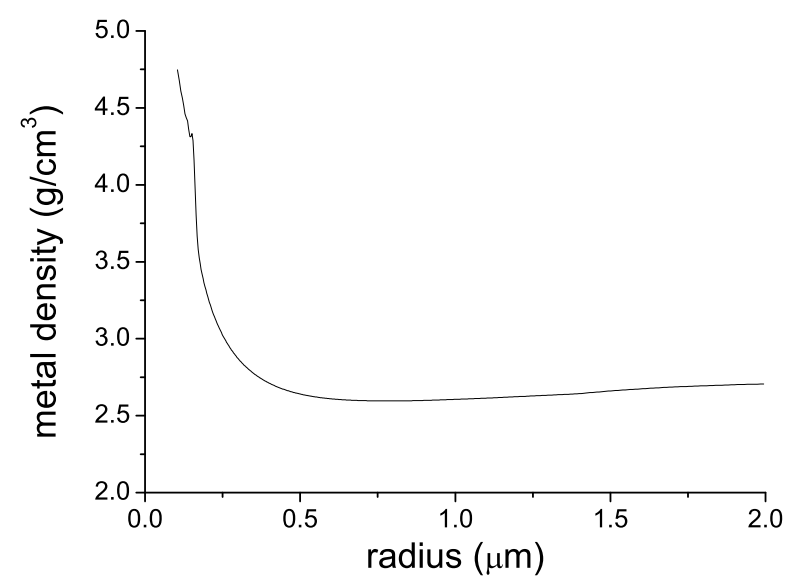

(b)

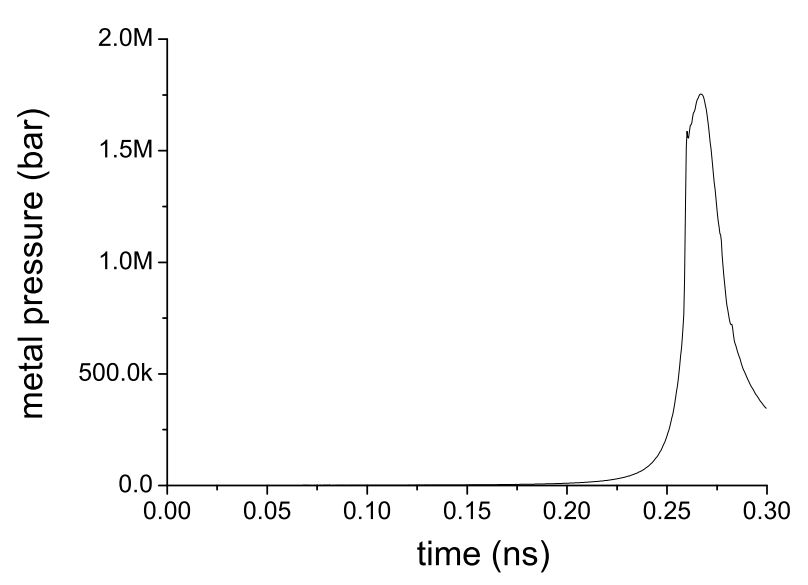

(d)

FIG. 9: (a) Density profile of metal at stagnation and the time evolutions of (b) density, (c) temperature and (d) pressure of the metal layer just outside the gas bubble. 


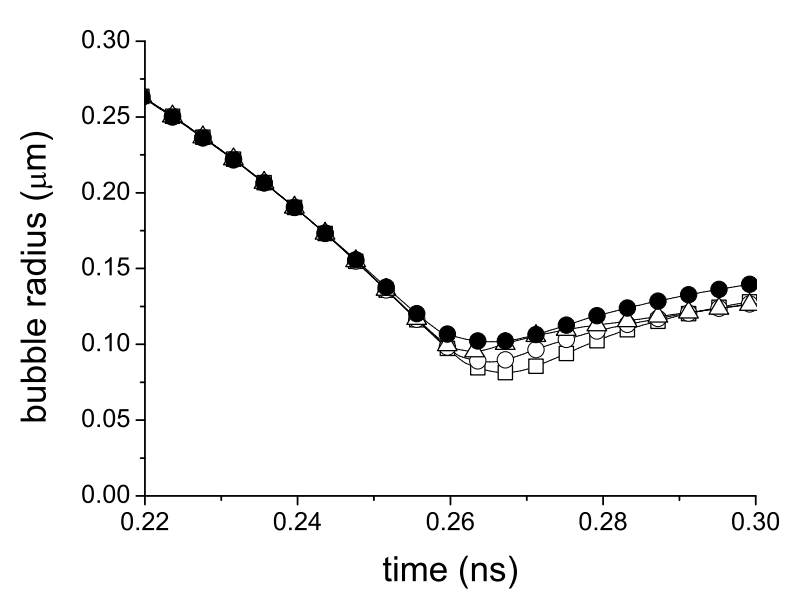

(a)

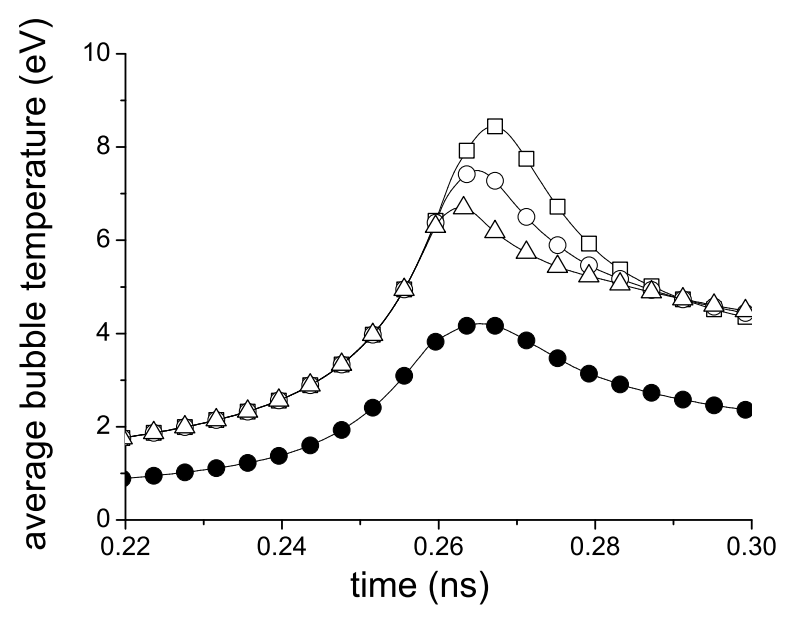

(c)

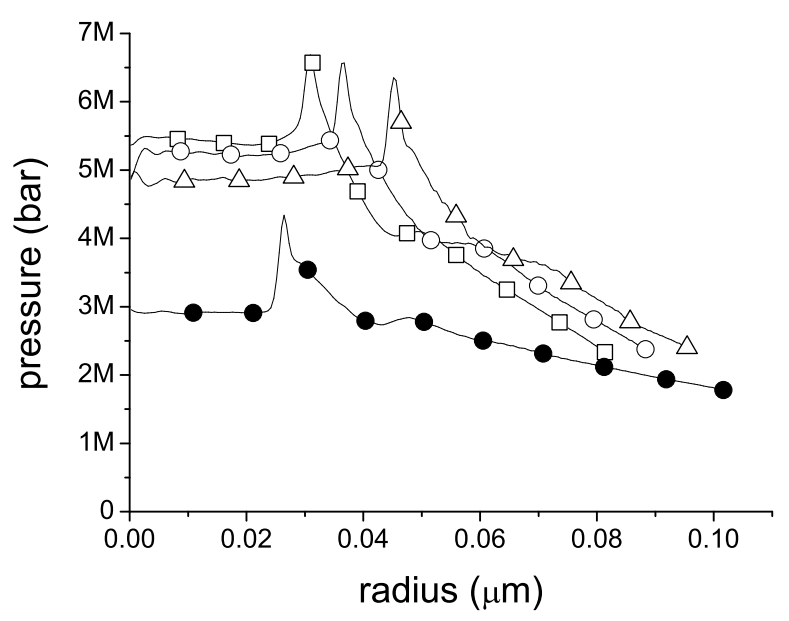

(b)

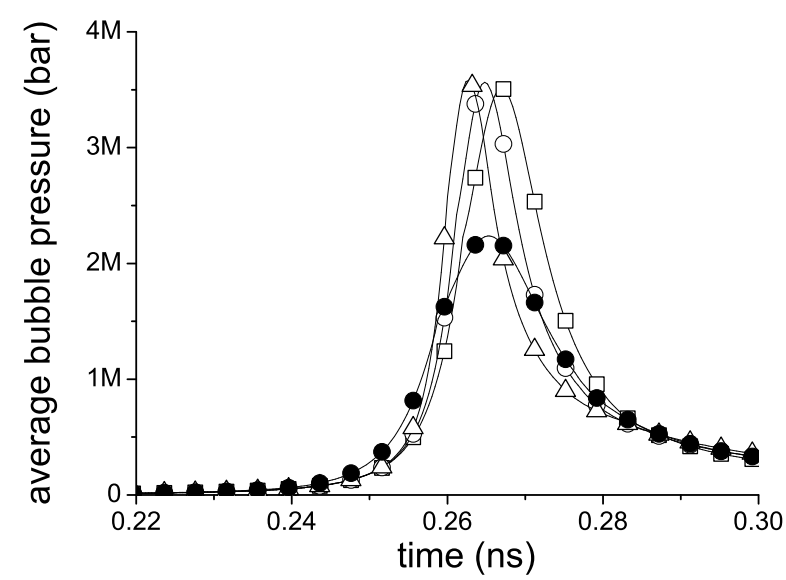

(d)

FIG. 10: (a) Time evolution of the bubble radius, (b) pressure profile inside the bubble at stagnation point, time evolution of (c) the average temperature and (d) the average pressure of the whole bubble. The gas content obeys IPEOS with $n=6$ (empty square); $n=9$ (empty circle); $n=12$ (empty triangle); and QEOS (solid circle). 


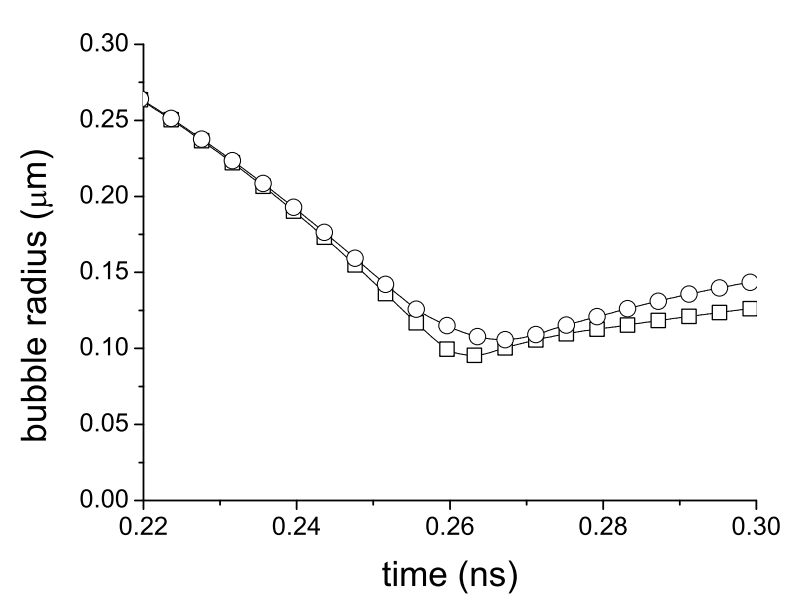

(a)

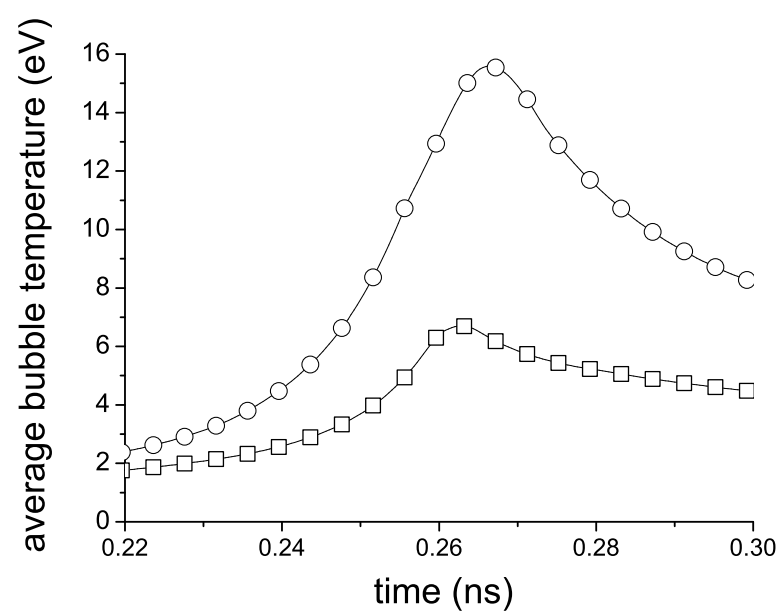

(c)

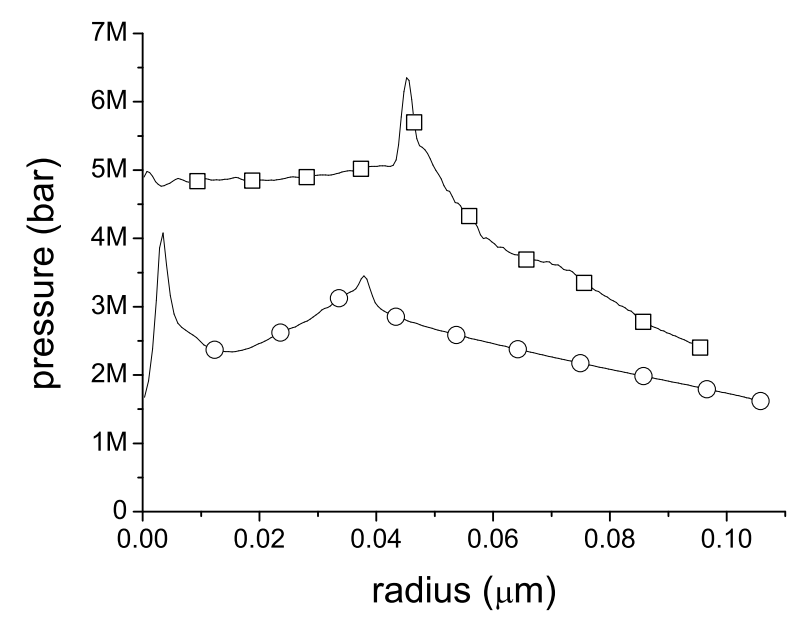

(b)

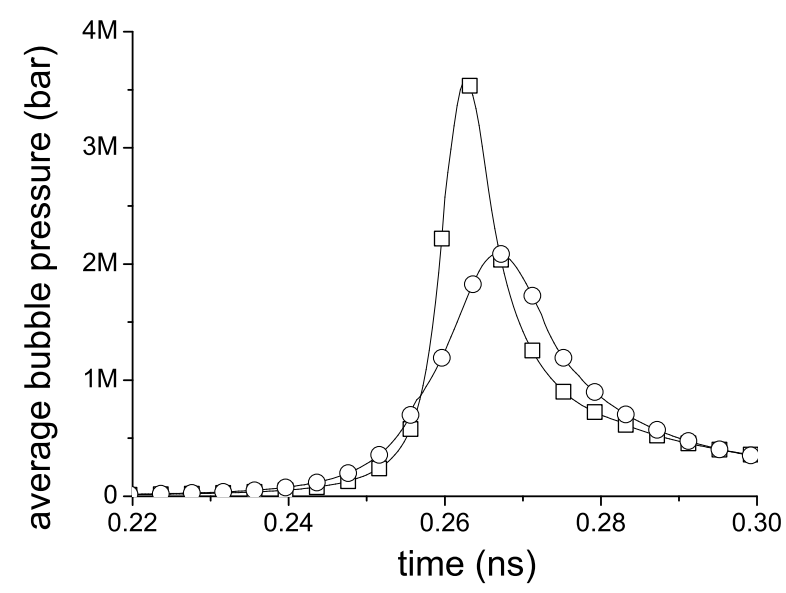

(d)

FIG. 11: (a) Time evolution of the bubble radius, (b) pressure profile inside the bubble at stagnation point, time evolution of (c) the average temperature and (d) the average pressure of the gas bubble. The gas content obeys IPEOS with $n=12$. Squares (circles) represent data obtained from simulations where effects of ionization are considered (ignored) . 


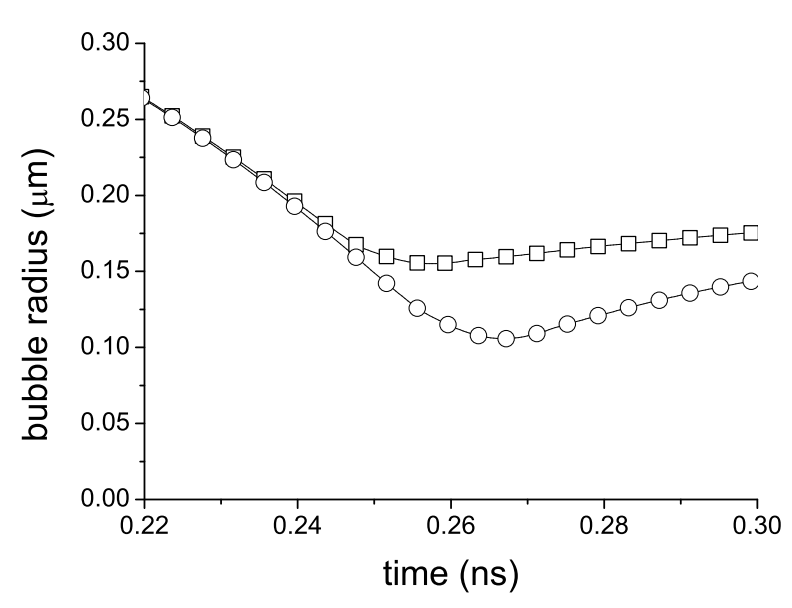

(a)

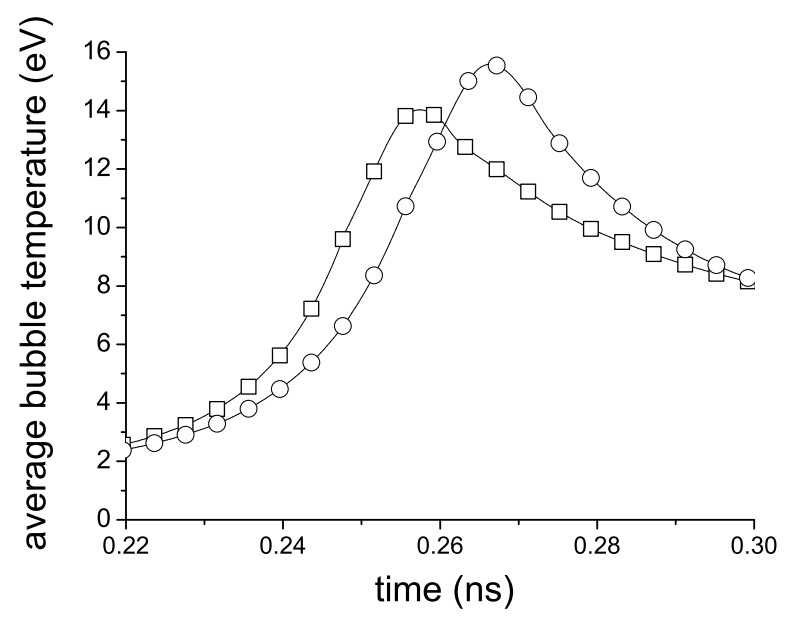

(c)

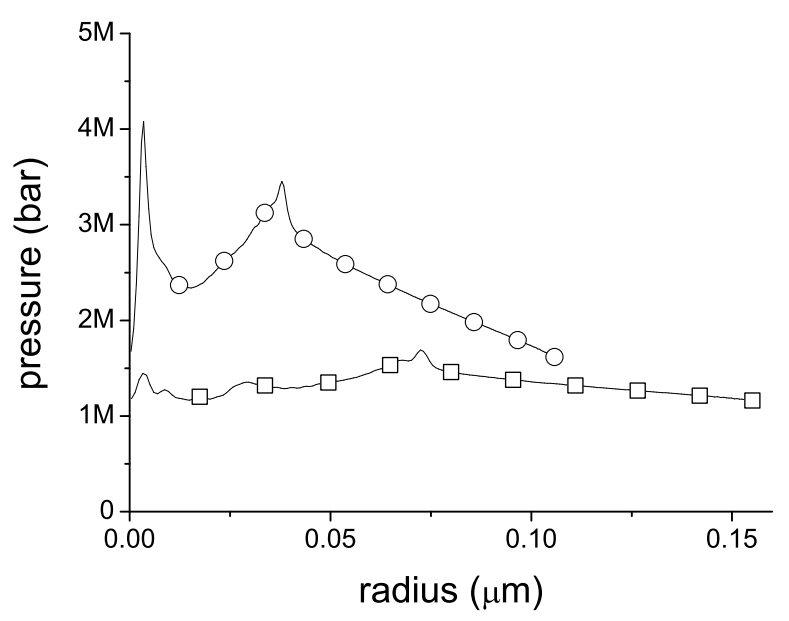

(b)

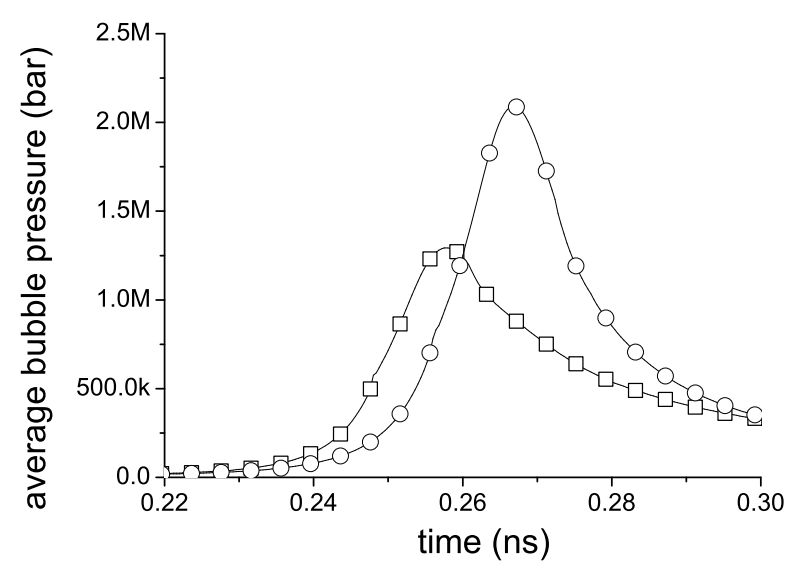

(d)

FIG. 12: (a) Time evolutions of bubble radius, (b) pressure profiles inside the bubble at stagnation point, time evolutions of (c) average temperature and (d) average pressure of the gas bubble. The gas content obeys VDW-EOS (square); and IPEOS $(n=12)$ without ionization (circle). 


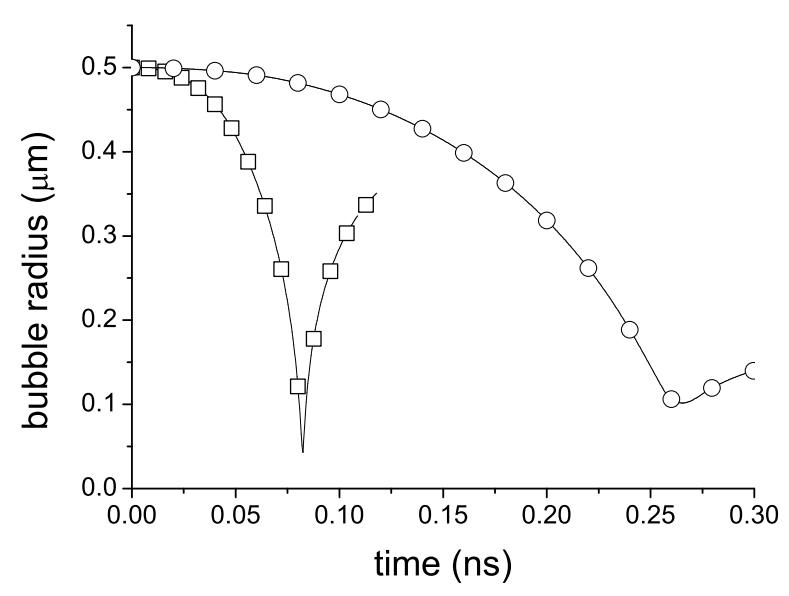

(a)

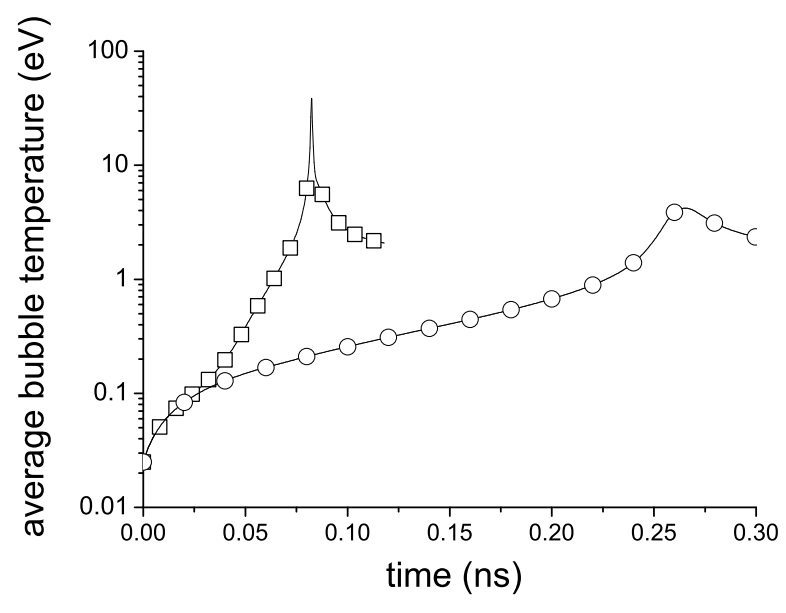

(c)

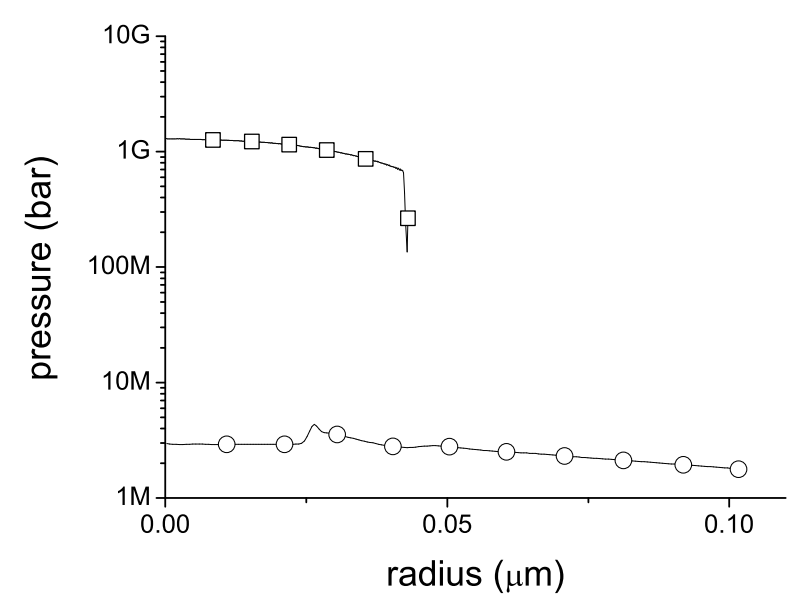

(b)

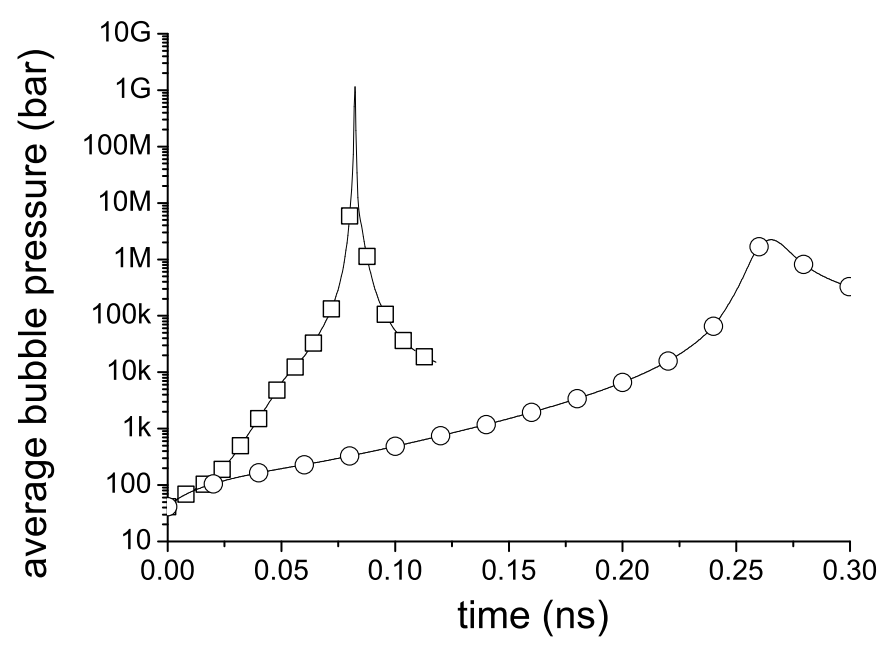

(d)

FIG. 13: (a) Time evolutions of bubble radius, (b) pressure profiles inside the bubble at stagnation point, time evolutions of (c) average temperature and (d) average pressure of the gas bubble. The metal surrounding obeys VDW-EOS (square); and GEOS (circle). 


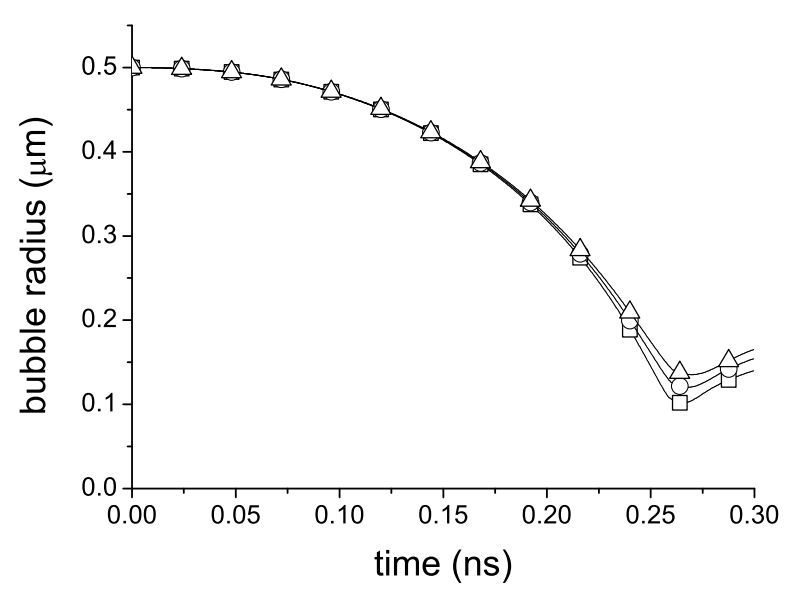

(a)

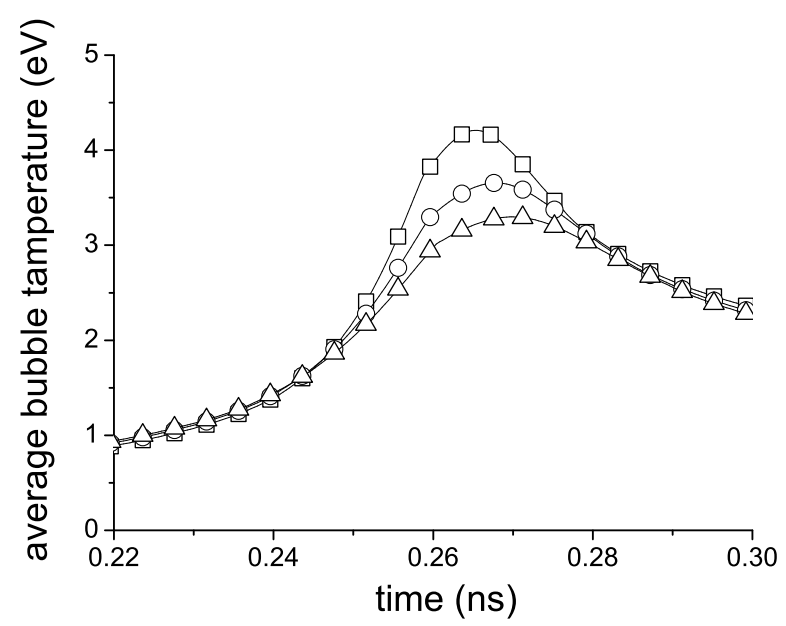

(c)

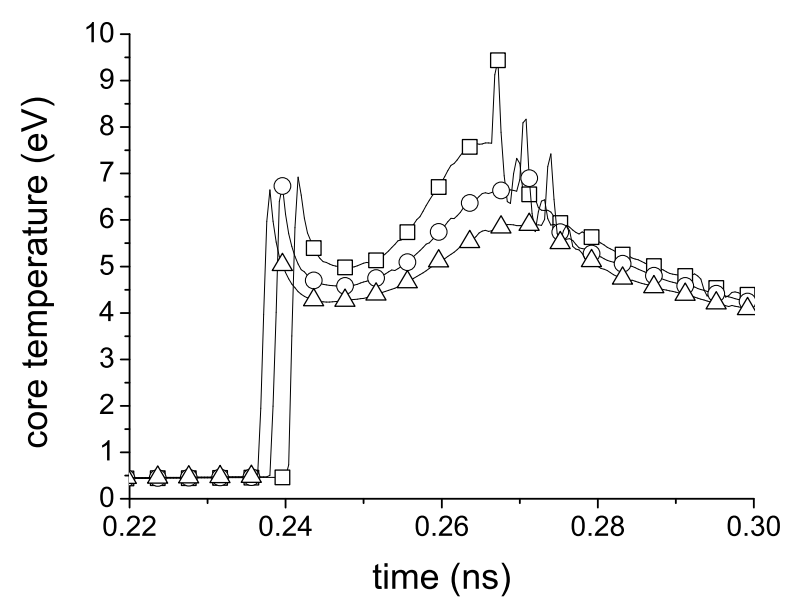

(e)

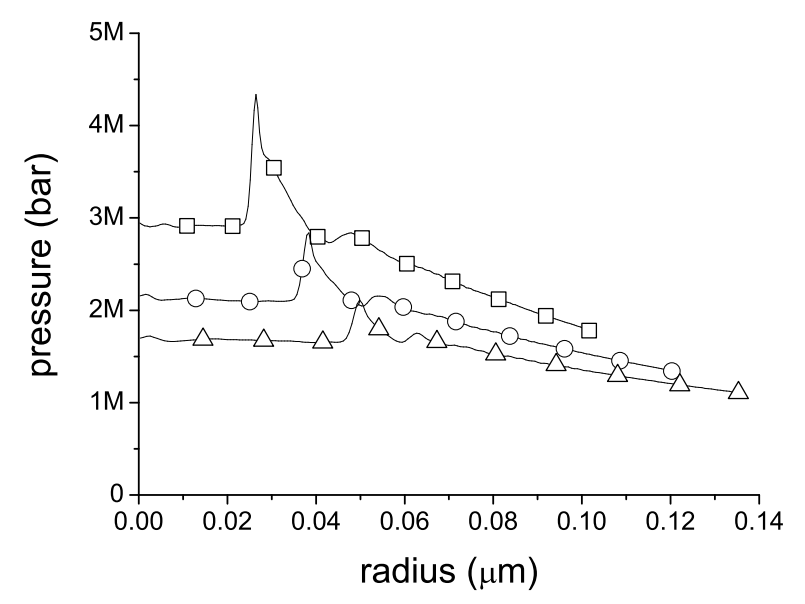

(b)

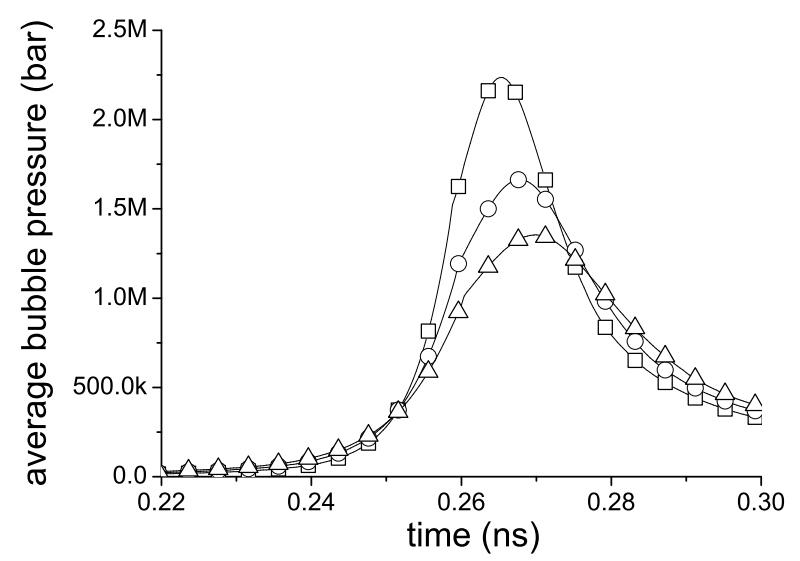

(d)

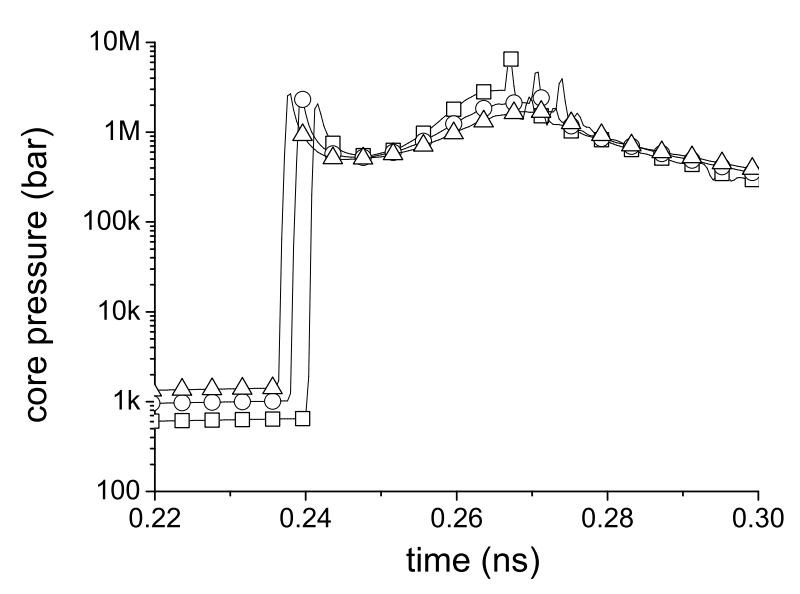

(f)

FIG. 14: (a) Time evolutions of bubble radius, (b) pressure profiles inside the bubble at stagnation point, time evolutions of (c) average temperature, (d) average pressure of the whole bubble, (e) core temperature and (f) core pressure for initial gas densities of $0.04 \mathrm{~g} / \mathrm{cm}^{3}$ (square), $0.06 \mathrm{~g} / \mathrm{cm}^{3}$ (circle) and $0.08 \mathrm{~g} / \mathrm{cm}^{3}$ (triangle) . 


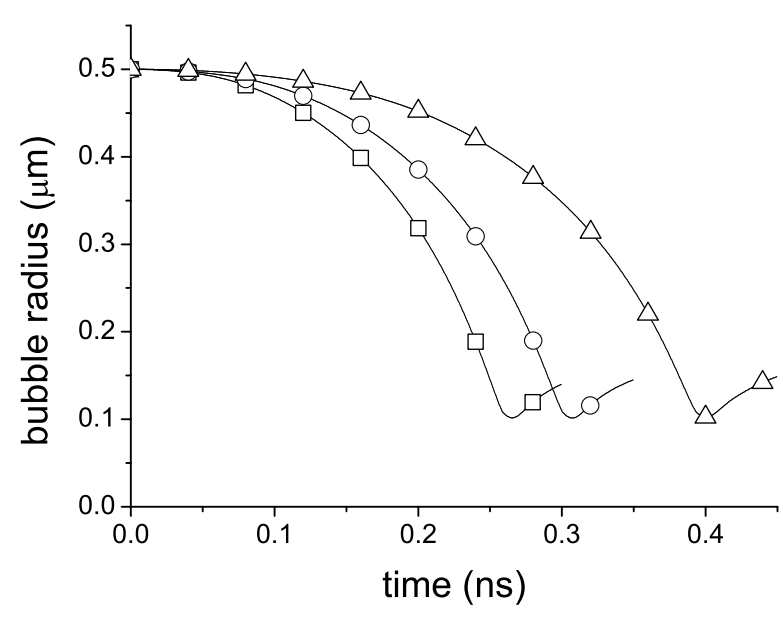

(a)

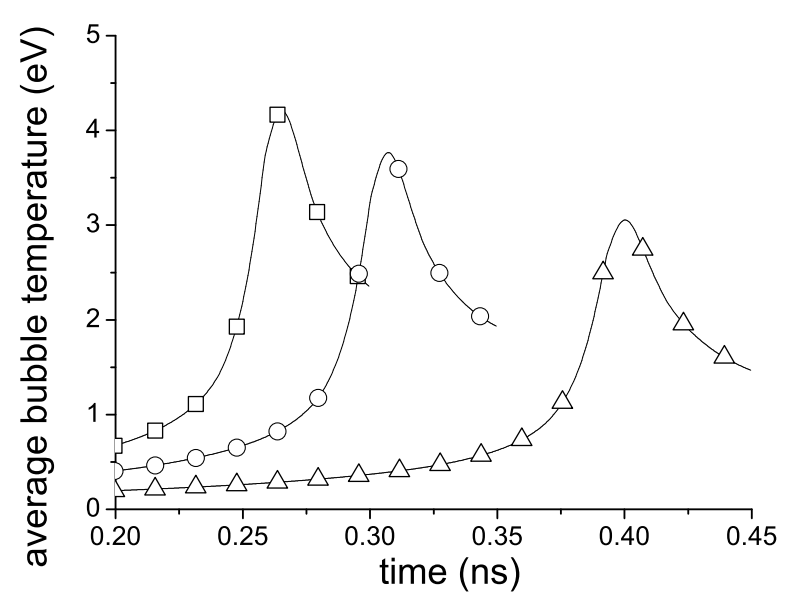

(c)

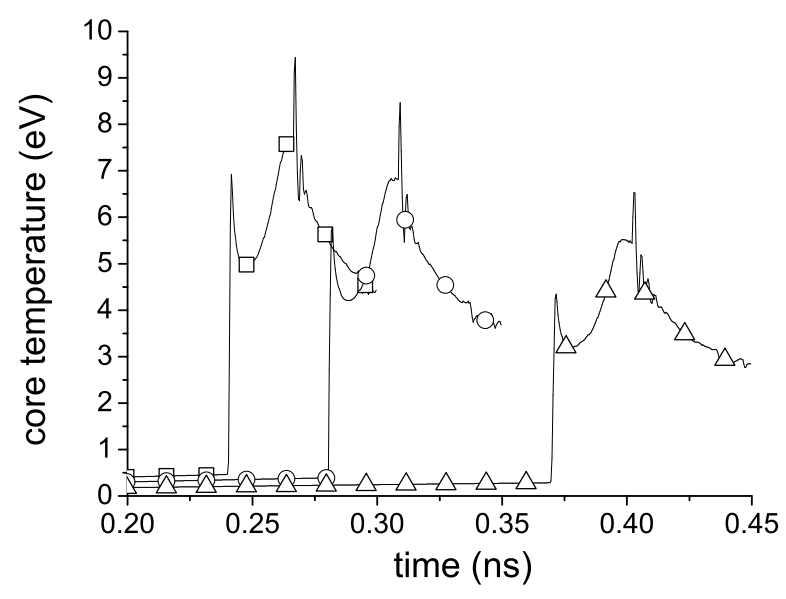

(e)

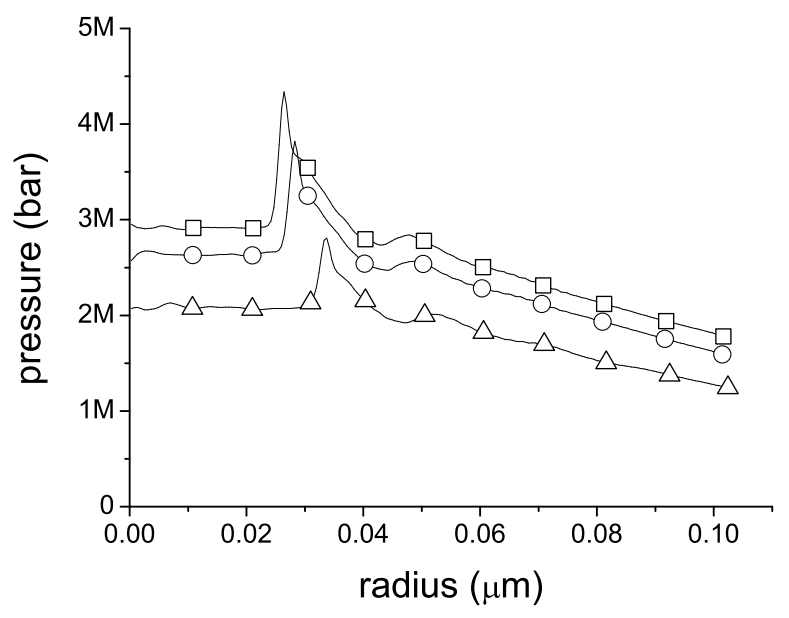

(b)

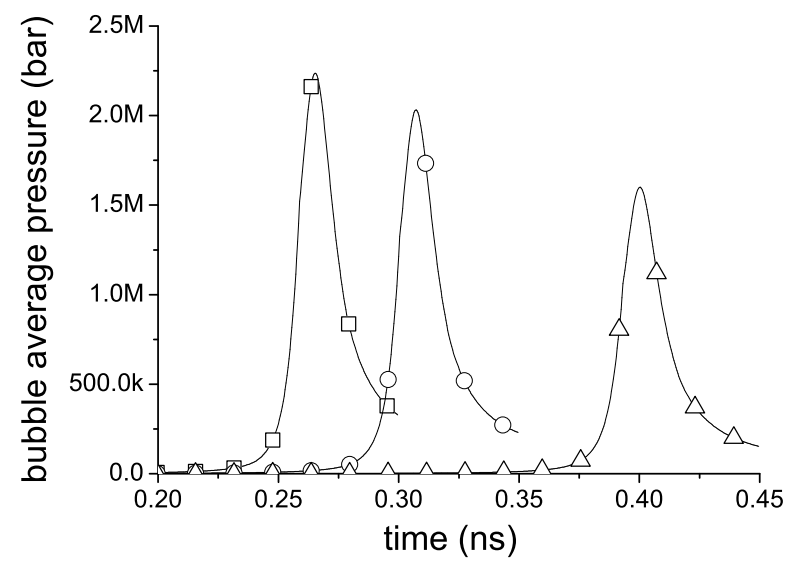

(d)

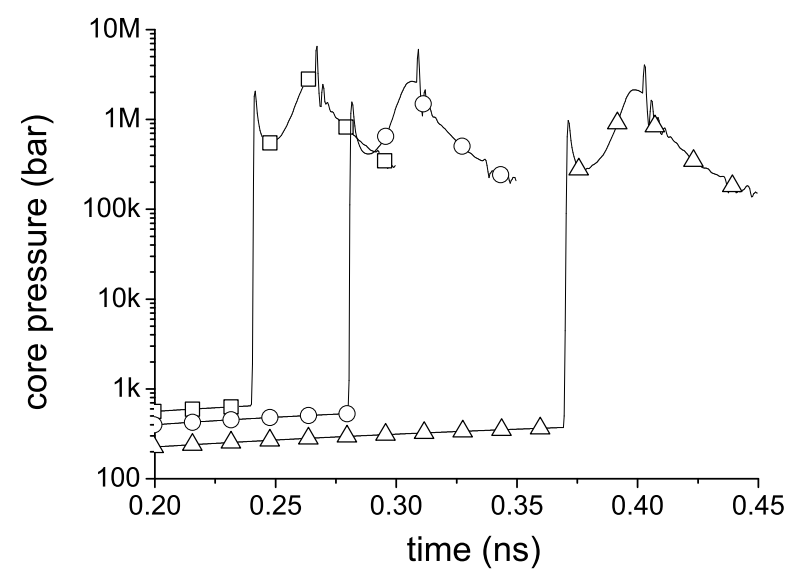

(f)

FIG. 15: (a) Time evolutions of bubble radius, (b) pressure profiles inside the bubble at stagnation point, time evolutions of (c) average temperature, (d) average pressure of the whole bubble, (e) core temperature and (f) core pressure for ion energy deposition rates of $15 \mathrm{~kJ} / \mathrm{g} / \mathrm{ns}$ (square), $10 \mathrm{~kJ} / \mathrm{g} / \mathrm{ns}$ (circle) and $5 \mathrm{~kJ} / \mathrm{g} / \mathrm{hs}$ (triangle). 